\title{
Competitive Imperfect Price Discrimination and Market Power*
}

\author{
Paul Belleflammeł Wing Man Wynne Lam; Wouter Vergote ${ }^{\S}$
}

\begin{abstract}
Two duopolists compete in price on the market for a homogeneous product. They can 'profile' consumers, i.e., identify their valuations with some probability. If both firms can profile consumers but with different abilities, then they achieve positive expected profits at equilibrium. This provides a rationale for firms to (partially and unequally) share data about consumers, or for data brokers to sell different customer analytics to competing firms. Consumers prefer that both firms profile exactly the same set of consumers, or that only one firm profiles consumers, as this entails marginal cost pricing (so does a policy requiring list prices to be public). Otherwise, more protective privacy regulations have ambiguous effects on consumer surplus.
\end{abstract}

${ }^{*}$ We are very grateful for comments from the senior editor, Ganesh Iyer, the associate editor and 2 anonymous referees. We thank Juanjo Ganuza, Axel Gautier, Yassine Lefouili, Johannes Johnen, Bruno Jullien, Kai-Uwe Kuhn, Jose-Luis Moraga Gonzalez, David Myatt, Allesandro Pavan, Martin Peitz, and participants to seminars and workshops in Alghero, Barcelona, Brussels, Louvain-la-Neuve, Marseille, Montpellier, Nancy, Norwich, Paris, Philadelphia, Stony Brook, Toulouse and Würzburg for useful comments and suggestions on earlier drafts. Wouter Vergote gratefully acknowledges financial support from the Belgian French speaking community ARC project \#15/20-072, Université Saint-Louis - Bruxelles, October 2015 - September 2020. Part of this research was performed when Paul Belleflamme was attached to Aix-Marseille School of Economics and Kedge Business School and Wouter Vergote was attached to the Université Saint-Louis Bruxelles; the support of these three institutions is gratefully acknowledged. A previous version of this paper circulated under the title 'Price Discrimination and Dispersion under Asymmetric Profiling of Consumers'.

${ }^{\dagger}$ CORE/LIDAM and Louvain School of Management, Université catholique de Louvain, 34 Voie du Roman Pays, 1348 Louvain-la-Neuve, Belgium, Paul.Belleflamme@uclouvain.be; also affiliated with CESifo.

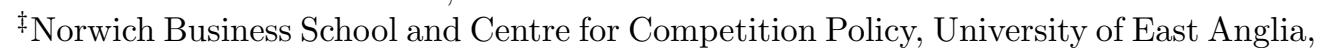
Norwich Research Park, NR4 7TJ, Norwich, United Kingdom, wing.m.lam@uea.ac.uk

${ }^{\S}$ Columbia University, Economics Department, 1022 IAB, 420 West 118th Street New York, NY 10027, wrv13@columbia.edu 
KEYWORDS: Price discrimination, price dispersion, Bertrand competition, privacy, big data.

\section{Introduction}

Context. Advances in digital technology have greatly facilitated firms' access to detailed information on consumers' willingness to pay. This has improved their ability to offer personalized prices and, potentially, to raise their profitability. Shiller (2014) provides empirical evidence that web browsing data gives firms more information on consumer willingness to pay compared to 'old school' demographic data, which increases profits by around $12.2 \%$. Similarly, a report of McKinsey\&Company (2016) shows that finer data analytics has improved customer segmentation and firm's profitability, while Mikians et al. (2012) empirically demonstrate the existence of personalized pricing. In addition, firms are also more open nowadays regarding their use of differential pricing strategies ${ }^{1}$ On the other hand, anecdotal evidence suggests that consumers are not always aware that other prices may be available to them, or that even if they are aware, it may be (too) costly to obtain access to a lower price. This paves the way for firms to price discriminate some consumers against others by selling the same good at different prices to different consumers.

Against this backdrop, we want to revisit price competition in data-rich environments where firms are able to profile customers and potentially differ in their ability to do so. Differences in abilities to profile consumers could come from different capacities to collect and analyze data (think of Amazon or Alibaba vs smaller online shops). ${ }^{2}$ This could also be due to having obtained different customer analytics from data collecting and processing companies, so-called 'data brokers' ${ }^{3}$

\footnotetext{
${ }^{1}$ For instance, Ant Financial Services Group (the financing unit of Alibaba) announced in 2015 the launch of Sesame Credit, a credit-scoring service that leverages big data and customer behavior analytics to calculate personalized interest rates for micro loans or personalized premiums for insurance services; see Business Wire (2015). See also European Insurance and Occupational Pensions Authority (2019) for a review of how motor and health insurance companies use big data for pricing.

${ }^{2}$ BenMark et al. (2017) describe the expertise that firms must develop to be effective in applying differential-pricing solutions.

${ }^{3}$ One such data broker is the Belgian Realdolmen, which clearly details on its website that it offers 'tailored' data services (see http://www.realdolmen.com/tactical-ict/ ict-trends/big-data, accessed on January 25, 2018). Interestingly, Realdolmen is partially controlled by Belgian supermarket chain Colruyt. One may speculate that Colruyt, given its privileged relationship with Realdolmen, may have an advantage when it comes to obtaining information about potential consumers compared to other companies, includ-
} 
More precisely, we study a model of price competition between firms in a homogenous good setting. Thanks to a clever use of big data, firms are able to 'profile' consumers, i.e., to identify their willingness to pay. Yet, they can only do this imperfectly: there is always a positive probability that any particular consumer will remain anonymous. This may be due to the available data being not sufficiently precise, or to consumers acting to protect their privacy ${ }^{4}$ Firms then charge a personalized price to those consumers that they are able to profile, while setting a 'uniform' price for all consumers who remain anonymous. Consumers may observe that firms charge different prices, but it is assumed that they can only buy at the price they have been individually offered.

We assume that firms have correlated abilities to profile consumers, which makes sense if they both use the services of the same data broker or if they share the data that one of them collected. In this setting, we address the following questions: Is being able to profile consumers enough to obtain market power through price discrimination in a duopolistic setting? If the firms' profiling ability depends on the data they need to purchase from a data broker, would this data broker sell these data exclusively to one firm? Is an improved profiling ability good or bad news for consumers? If consumers would have the option to hide their characteristics, would they like to do so? If uniform prices were observable ('list prices') would this lead to more competition?

Main results. Without the possibility to price discriminate, price competition in uniform prices would result in marginal cost pricing, the classical Bertrand Paradox. Yet, having the ability to profile consumers does not automatically translate into market power. It only does so when both firms have the ability to profile consumers but they do not always profile the same consumers. The mechanism that yields market power in our model relies on the strategic effect of the firms' uniform prices (set in stage 1) on both firms' personalized prices. Indeed, there will be a positive probability that the better informed firm recognizes consumers the other firm does not and this firm can thus guarantee positive profits as long as the uniform price of the less informed firm is above the marginal cost. Hence, when the latter increases its uniform price, competitive pressure on 'profiled' consumers de-

ing its competitors. This advantage may translate into asymmetric abilities to profile and target consumers.

${ }^{4}$ Consumers can use obfuscation strategies such as clearing cookies from their browsers, logging off their Google and Facebook accounts, or adopting proxy servers or ad-blockers. In our baseline model, we take such hiding as exogenous; in Section 5 , we discuss how our results could be affected if hiding was endogenized. 
creases. Therefore, the less-informed firm will always have an incentive to set a uniform price above its cost, yielding a price equilibrium that displays market power. This mechanism breaks down in two scenarios: when both firms always profile the exact same set of consumers and when the less informed firm can never profile any consumer. In these two cases, the only equilibrium is the Bertrand paradox, where both firms charge all consumers a price equal to marginal cost (irrespective of consumers being profiled or not). Hence, having the ability to price discriminate against profiled consumers does not necessarily imply market power.

Yet, our analysis also indicates that firms are likely to escape these situations in which the Bertrand Paradox continues to prevail. It is indeed in the interest of any data provider to make sure that both firms are able to profile consumers, albeit with different abilities to do so. If data about consumers is collected by one of the firms, our results show that this firm has an incentive to share part of this data with its competitor, so as to relax price competition. If data brokers are the data providers, they will find it optimal to provide data services of different 'quality' (in terms of profiling ability) to the two firms. That is, data brokers will 'tailor' their data services in a non-exclusive way, not to satisfy the 'needs' of the firms that demand data services, but rather to soften price competition in the product market and, thereby, increase the firms' willingness to pay for their services.

We also assess how competitive differential pricing affects consumers. Obviously, consumers are hurt as soon as firms are both able to profile them but in differentiated ways, as equilibrium prices are then set above marginal cost. The more interesting question is how consumers are affected when the firms' profiling abilities are improved or become more similar. To address this question, we focus on the simpler case of perfectly correlated 'profiling technologies'; in this case, any consumer profiled by the so-called 'less-informed' firm is also profiled by the so-called 'better-informed' firm, but the reverse is not true. In this setting, we provide a set of comparative statics results with respect to the precision of the profiling technology and the degree of asymmetry between firms' profiling technologies. We show that increasing the precision of profiling technologies increases both uniform and personalized prices, leading to instances in which some profiled consumers are right to fear that they are being priced closer to their 'pain point' compared to the uniform price they would receive if they would be able to hide. This provides incentives to hide so as to avoid excessive prices, i.e., there is a demand for privacy. However, some consumers may gain from improved profiling, namely those consumers with a low valuation, who start purchasing (and enjoying a positive surplus) when they are no longer anonymous; as what they gain may outweigh what other consumers lose, improved profiling may lead to an 
overall increase in consumer surplus.

We then show that reducing the asymmetry between firms' profiling technologies has a non-monotone effect on the uniform prices. More specifically, uniform prices are at their highest level for an intermediate level of asymmetry $5^{5}$ We also establish that for given uniform prices, personalized prices decrease as firms' profiling technologies become more symmetric, which is due to the higher intensity of competition between firms for consumers profiled by both firms. This has interesting implications for consumer surplus. For relatively high levels of symmetry between firms' profiling technologies, further increasing the symmetry benefits the consumers as it leads to both lower uniform prices and lower personalized prices. For relatively low levels of symmetry, however, increasing the symmetry leads to higher uniform prices but lower personalized prices.

We finally confirm that when consumers can always purchase the product at the 'list' price, competitive pressure is higher. The intuition is simple: a firm that wants to price discriminate does not just compete against the prices of its competitor but also against its own uniform price. The effect is very stark in our model: marginal cost pricing prevails again at the subgameperfect equilibrium, bringing us back in the Bertrand Paradox. Firms thus have incentives to prevent consumers from having access to multiple prices. In addition, we show in a simplified version of our model in which consumers can only be of two types, that no other (mixed strategy) subgame-perfect equilibrium exists.

Related literature. Price competition with imperfect profiling has rarely been studied in an oligopoly setting before. Either imperfect price discrimination is considered in a monopoly setting, as done recently by Belleflamme and Vergote (2016), and Valletti and Wu (2016). Or oligopolistic competition is introduced, but with restricted discrimination abilities for the competing firms. Seminal papers are Thisse and Vives (1988), Corts (1998), Liu and Serfes (2004), Encaoua and Hollander (2007); see also Taylor and Wagman (2014) for a synthesis, Kehoe et al. (2018) for an analysis of the impacts of big data on price competition in an oligopoly market for branded experience goods, and Kim et al. (2019) for an analysis of the effects of consumer profiling on merger analysis. In this literature, the choice given to firms is often between the two extremes of perfect discrimination and uniform pricing; when third-degree price discrimination (i.e., group pricing) is considered, the focus is on symmetric situations where firms have the same ability to discriminate. One exception is Chen and Iyer (2002), who study price competition under

\footnotetext{
${ }^{5}$ This result, which holds generally, contributes to explain why firms are willing to share data and data brokers to tailor their services.
} 
imperfect consumer addressability and establish price dispersion in equilibrium. This paper differs from Chen and Iyer (2002) in two ways. First, we fully characterize the two stage price competition equilibrium with uniform price and personalized price. ${ }^{6}$ Second, the strategic uncertainty that firms face come from a different source: in Chen and Iyer (2002), imperfect addressability makes each firm uncertain as to whether it is competing with the other firm or not (similar to Varian (1980), and Burdett and Judd (1983)), whereas in our paper, imperfect profiling makes each firm not sure about the pricing strategy of the other firm, while knowing that competition is always present. Our paper is also related to classical papers on targeted advertising such as Shaffer and Zhang (2002) and Iyer et al. (2005). In these papers, firms have perfect information about consumers' preferences and decide on which segment of consumers to target their advertisement. Asymmetric targeting arises as part of the equilibrium to limit the intensity of price competition, whilst firms have the same ability to target. Our paper, however, allows firms to differ in their ability to profile and focus on varying the intensity of price discrimination 7

Our paper also provides some important insights into the debate on privacy regulation. An early contribution, Calzolari and Pavan $(2006)$, focuses on the incentives of firms to share private information about consumers. More recently, the literature on targeting and privacy has grown considerably, triggered by advances in digital tracking; see Acquisti et al. (2016) for a survey. However, this literature focuses on independent targeting, whereas we study correlated targeting. Also, by covering the whole spectrum from no discrimination to perfect discrimination, and by letting firms differ in their intensity of price discrimination, we are able to shed new light on issues related to privacy.

This paper further relates to the literature that studies the incentives of data brokers. For example, Braulin and Valletti (2016) and Montes et al. (2019) also consider competitive targeting. However, there are two important differences. First, we consider homogeneous products, whereas they consider differentiated products. Second, we examine the mixed strategies equilibria under different 'degrees' of asymmetry and correlation in profiling technologies, but they focus on the pure strategies equilibria under perfectly asym-

\footnotetext{
6 Chen and Iyer (2002) mainly characterize the price competition equilibrium in personalized prices. They briefly discuss price competition in both uniform price and personalized price in Section 5, but with consumers observing both uniform prices and personalized prices.

${ }^{7}$ Moreover, our model differs with respect to discount and poaching models (e.g., VillasBoas (1999)), as firms here can set personalized prices above the previously set uniform price.
} 
metric profiling technologies (i.e., one firm is unable to profile consumers). When products are differentiated vertically (Braulin and Valletti (2016) $)$ and horizontally (Montes et al. (2019)), they find that the data broker finds it profitable to sell its data exclusively to only one firm. We, however, show that the ability to profile is irrelevant to market power if firms' profiling technologies are fully symmetric (and perfectly correlated) or fully asymmetric, as the Bertrand paradox prevails in both cases. Because of this, we find that in contrast to the result of Braulin and Valletti (2016) and Montes et al. (2019), the data broker may have incentives to sell its data to all competing firms, as long as firms end up with different abilities to profile consumers. Another example is Jentzsch et al. (2013), who study price discrimination in two dimensions (group and individual price discrimination) in a Hotelling model. However, they do not study the effect of imperfect profiling; that is, in their model, each firm knows its rival's information set. Therefore, price discrimination arises in equilibrium but not price dispersion, though they also find that partial information sharing is profitable for firms.

The rest of the paper is organized as follows. In Section 2, we present our modeling framework. In Section 3 , we solve the model for its subgame-perfect equilibrium and explain why competitive differential pricing may relax competition. We then derive the implications of our results for data markets (Section 4) and for consumers (Section 5). Finally, in Section 6, we draw some policy and empirical implications from our analysis.

\section{The model}

We consider a market where two firms produce a homogeneous product at a constant marginal cost, which is set to zero for simplicity. We describe in turn the main assumptions regarding consumers and firms, as well as the timing of the game.

Consumers. There is a unit mass of consumers who vary in the valuation that they attach to the homogeneous product. The valuation of consumer $x$ is noted $r(x)$, which is randomly and independently drawn according to the distribution function $F():. \mathbb{R} \rightarrow[0,1]$ with support $[0, \bar{r}]$, and with associated continuously differentiable density function $f():. \mathbb{R} \rightarrow \mathbb{R}$. Consumers wish to purchase at most one unit of the product and they do so from the firm that offers the lowest price (we say more on this below). Noting this price $p$, we can express the expected demand from consumer $x$ at $p$ as the probability that the consumer has a valuation that is at least as large as $p$, i.e. $\operatorname{prob}(r(x)>p)$. This probability is given by the survival function $S():. \mathbb{R} \rightarrow[0,1]$ where 
$S(p)=1-F(p)$. Since we assume that the mass of consumers is equal to 1 and that valuations are drawn independently, the survival function also represents the aggregate expected demand (referred to hereafter as the 'demand'). We impose the additional assumption that demand is log-concave: $S^{\prime}(p) / S(p)=$ $-h(p)$ is non-increasing in $p$, where $h(p)$ is the hazard rate.

Firms. Each firm has access to a 'profiling technology' that allows it to identify the valuation of a consumer probabilistically. When a firm identifies the valuation of a consumer, it is in a position to price discriminate and charge this consumer a personalized price. For the consumers that a firm does not profile, the firm sets a uniform price.

Without loss of generality, assume that the probability of identifying the valuation of a consumer is equal to $\lambda_{A}=\lambda \in[0,1]$ for firm $A$ and $\lambda_{B}=\beta \lambda$ for firm $B$, where $0 \leq \beta \leq 1$. The parameter $\lambda$ can be interpreted as a measure of firm $A$ 's ability to profile consumers. Alternatively, $1-\lambda$ can be interpreted as the capacity of consumers to hide from firm $A$. As for the parameter $\beta$, it measures the degree of symmetry between the firms' profiling technologies: if $\beta=1$, firms have symmetric technologies (they are 'equally' informed); for any $\beta<1$, firm $B$ 's is at a disadvantage with respect to firm $A$ in terms of profiling consumers (firm $A$ is the 'better-informed' firm and firm $B$, the 'less-informed' firm).

Let us now determine the probabilities for a given consumer $x$ to be profiled by both firms, only one of them, or none of them. To this end, we denote by $a$ (resp. $b$ ) the conditional probability that firm $A$ (resp. $B$ ) profiles any consumer $x$ in the event that firm $B$ (resp. $A$ ) does so as well. To allow for any degree of (positive) correlation between these two conditional probabilities, we define $a=\phi \lambda$ and $b=\phi \beta \lambda$, where $\left.\phi \in\left[1, \frac{1}{\lambda}\right]\right]^{8}$ The parameter $\phi$ is a measure of the correlation of the profiling technologies: the higher its value, the more correlated the technologies. At the lower bound $(\phi=1)$, the profiling technologies are independent, while at the upper bound $\left(\phi=\frac{1}{\lambda}\right)$, the technologies are perfectly correlated (i.e., firm $A$ 's technology profiles any consumer that firm $B$ profiles as well, but not vice versa).

We can now compute the jointly correlated profiling probabilities for a given consumer. Let $M_{2}$ denote the probability that both firms profile the consumer, $M_{K}$ the probability that only firm $K$ profiles the consumer $(K=A, B)$, and $M_{0}$ the probability that no firm profiles the consumer. Using the previous definitions, we have $9^{9} M_{2}=a \lambda_{B}=b \lambda_{A}=\beta \phi \lambda^{2}$,

\footnotetext{
${ }^{8}$ We would in general expect that the profiling technologies of firms are correlated: when one firm profiles a consumer, it increases the chances that the other firm will do so as well.

${ }^{9}$ The conditions $\lambda, \beta \in[0,1]$ and $\phi \in\left[1, \frac{1}{\lambda}\right]$ ensure that all four probabilities are between
} 
$M_{A}=(1-b) \lambda_{A}=\lambda(1-\beta \phi \lambda), M_{B}=(1-a) \lambda_{B}=\beta \lambda(1-\phi \lambda)$, and $M_{0}=1-\left(M_{2}+M_{A}+M_{B}\right)=1-\lambda-\beta \lambda(1-\phi \lambda)$.

As we set the total a mass of consumers equal to unity, the latter probabilities can also be seen as the respective masses of consumers in four distinct market segments. To ease the exposition, we name these segments as follows: the 'transparent' segment (with mass $M_{2}$ ) comprises those consumers that both firms have profiled; the ' $K$-translucent' segment (with mass $M_{K}$ ) comprises those consumers that only firm $K$ has profiled; the 'opaque' segment (with mass $M_{0}$ ) comprises those consumers that no firm has profiled. For future reference, we note that there are three particular instances in which the $B$-translucent segment is empty: (i) $\beta=0$ (firm $B$ cannot profile any consumer; in this case, we also have $M_{2}=0$ ), (ii) $\lambda=1$ (firm $A$ is able to profile all consumers; in this case, we also have $M_{0}=0$ ), (iii) $\phi=1 / \lambda$ (profiling technologies are perfectly correlated). As for the $A$-translucent segment, the only instance in which it is empty is when $\phi=1 / \lambda$ and $\beta=1$ (profiling technologies are perfectly correlated and equivalent).

Timing of the game. Before the game starts, the firms acquire their profiling technology, with respective precisions $\lambda_{A}$ and $\lambda_{B}$. The values $\left(\lambda_{A}, \lambda_{B}\right)$ are assumed to be common knowledge ${ }^{10}$ In the first stage of the game, firms set their uniform price $p_{K}$, with $K \in\{A, B\}$, for the consumers that they are not able to profile. In the second stage, after observing the uniform prices, firms set personalized prices for the consumers that their profiling technology allows them to profile; these prices are noted $p_{K}(x)$, with $x$ referring to the identity of the consumer with valuation $r(x)$. This assumption can be justified in several ways. First, a sequential price setting is commonly assumed in the literature (see Thisse and Vives (1988)). Second, it also reflects the relative flexibility in the adjustment of personalized prices compared to uniform prices (see, e.g., Choe et al. (2018)). Finally, as we show in the analysis, simultaneous pricing would lead firms back to the Bertrand paradox.

In the third stage, consumers decide whether or not to buy the product, and from which firm to buy after observing the price offered to them by each firm. We assume that consumers receive only one price offer from each firm, either a uniform or a personalized price. As a consequence, profiled consumers are not able to arbitrage between their personalized price and the uniform price. This assumption is reasonable if price offers are sent out electronically and if consumers have to incur a sufficiently high search cost

\footnotetext{
0 and 1.

${ }^{10}$ For now, we take the firms' profiling technologies as exogenous. In Section 4 we discuss how to endogenize the technologies by letting firms share datasets or by introducing data brokers in the game.
} 
to know about the uniform price. The fact that profiled consumers do not (or have a hard time to) observe uniform prices is not incompatible with the fact that firms do observe each other's uniform price. Firms may indeed infer the other firm's uniform price by using sophisticated technologies (such as robots) that are not accessible to consumers ${ }^{11}{ }^{12}$ We also need to set a couple of tie-breaking rules to decide how consumers choose when firms charge them the exact same price. For this, we assume that although consumers observe only one price from a given firm, they can determine whether this price is a personalized price or a uniform price. If both firms quote the same price but one price is personalized while the other is a uniform price, we assume that the consumer chooses the firm offering the personalized price. If both prices are the same and of the same nature (both personalized or both uniform), then we assume that the consumer chooses to purchase from each firm with equal probability.13

\footnotetext{
${ }^{11}$ This is a common assumption made in the literature. It has been justified in the brick-and-mortar world in which regular prices are viewed as more of a strategic variable than promotions. Arguably, this justification may seem less convincing for the online world that we consider in our paper (as prices can be changed more easily). However, it remains true in the online world that uniform (regular) prices are made public, while personalized prices are, by nature, only visible to the targeted consumers. We are thus confident that in the online world as well, the uniform price is a more long-term variable than the personalized prices (which have a more tactical role). It is also true that, even if uniform prices are published, they are harder to find for consumers than for firms (which have more sophisticated tools at their disposal). For instance, it appears that in the US, finding prices is very difficult for consumers in the health sector, even when firms have the legal obligation to publish prices (see Quito and Shendruk (2019)).

${ }^{12}$ The sequence of pricing decisions could also be justified as the equilibrium outcome of the following game. In the first stage, firms decide whether or not to commit to their uniform price before setting their personalized prices (commitment is achieved by publishing the price). In the second stage, firms set prices, not being able to change their uniform price if they committed to it in stage one. From the main results of our paper, it follows that, if the commitment costs are small enough, there are two pure-strategy subgame perfect Nash equilibria in this game: either both firms commit or none of them does. Clearly, the equilibrium in which both firms commit is Pareto-dominant and we can thus expect firms to find ways to coordinate on this equilibrium.

${ }^{13}$ There is evidence showing that consumers may favor either the personalized price or the uniform price. For instance, Wattal et al. (2012) find a negative effect of personalization in e-mails due to privacy intrusion, whereas Feld et al. (2013) find a positive effect of name personalization in direct mail. Using the other tie-breaking rule slightly complicates matters but should not change anything qualitatively: although the equilibrium price distribution of targeted prices will be different, they will generate the same expected payoff.
} 


\section{Why competitive differential pricing relaxes competition}

We establish here our main result: although firms compete in prices on the market for a homogeneous product, they obtain positive profits at equilibrium whenever they are both able to profile consumers but not the exact same set of consumers. In other words, competitive differential pricing allows, in general, the duopolists to escape the so-called Bertrand paradox.

Before establishing this result formally, let us discuss the intuition behind it. The major force driving the result is the uncertainty that the two firms face regarding the pricing strategy of their competitor. In particular, when one firm (say firm $A$ ) profiles some consumer, it is uncertain as to whether the other firm has profiled her as well or not. Firm $A$ knows that with probability $1-b$, firm $B$ did not profile this consumer (who belongs then to the $A$-translucent segment) and offered her its uniform price $p_{B}$; in that case, firm $A$ can win this consumer with an appropriate personalized price and assure itself positive profits. However, with probability $b$, firm $B$ did profile the consumer, which implies that both firms compete then for this consumer through personalized prices (we are then on the transparent segment) ${ }^{14}$

We show below that this uncertainty leads firms to set positive (i.e., above marginal cost) personalized prices, most often in a randomized way. This means that the second-stage equilibrium yields positive expected profits from profiling for both firms. What is key is that these expected profits increase with the uniform prices that firms set in the first stage of the game. The intuition is simple: as indicated above, a firm that profiles a consumer can secure a positive profit by undercutting the competitor's uniform price in the event that the competitor does not profile this consumer; hence, the higher the competitor's uniform price, the larger the expected profit.

Moving now to the first-stage of the game, it becomes clear that, anticipating what we just showed, firms will never set a uniform price equal to marginal cost (zero in this model); if they were, they would lose any chance to make profits from the consumers that they manage to profile. This completes the argument, as it proves that all prices (uniform and personalized) will be set above marginal cost.

Yet, we should not forget that firms can only charge their uniform price to the consumers that they fail to profile. Hence, the competition for these consumers drives uniform prices down (in the traditional Bertrand fashion).

\footnotetext{
${ }^{14}$ The same goes for any consumer that firm $B$ profiles, as this consumer could belong either to the $B$-translucent segment or to the transparent segment (with respective probabilities $1-a$ and $a$ ).
} 
When choosing its uniform price, each firm will thus balance two opposite inclinations: lower the price to be more competitive on the opaque segment and on the competitor's translucent segment, but raise it to relax competition on the other two segments (where it can set personalized prices). This tradeoff may lead firms to randomize uniform prices as well.

In short, the possibility to price discriminate together with uncertainty about the nature of price discrimination competition (did only one firm identify the consumer or did they both?), generates strictly positive prices and possible randomization of all prices. Let us now establish this result more formally.

\subsection{Personalized pricing equilibrium}

We first study the optimal personalized prices, given the observed uniform prices $\left(p_{A}, p_{B}\right)$. That is, we focus on the competition that takes place on the transparent segment (of mass $M_{2}=\beta \phi \lambda^{2}$ ) and the two translucent segments (of respective masses $M_{A}=\lambda(1-\beta \phi \lambda)$ and $M_{B}=\beta \lambda(1-\phi \lambda)$ ). In other words, firms choose their personalized prices $\left(p_{A}(x), p_{B}(x)\right)$ for the consumers that they-and possibly their competitor-have identified.

As a first step, we establish that if the uniform prices are equal to marginal cost, so are the personalized prices.

Lemma 1. If $\left(p_{A}, p_{B}\right)=(0,0)$, then $\left(p_{A}(x), p_{B}(x)\right)=(0,0)$ is the unique equilibrium at the second stage of the game.

Proof. Clearly, $p_{A}(x)=0$ is a (weakly) best reply to $p_{B}(x)=0$ and vice versa. Thus we can conclude that $\left(p_{A}(x), p_{B}(x)\right)=(0,0)$ is a Nash equilibrium. No other equilibrium in pure strategies can exist, since there would be a profitable deviation. Suppose that there exists an equilibrium in mixed strategies. The support of these strategies must contain only strictly positive prices (and be symmetric). But then, the upper limit of the support must have positive mass. If not, playing this price will yield an expected payoff of zero. Yet, for any point with positive mass in the support, there is an incentive to slightly undercut this price as to increase profit.

We now show that when at least one uniform price is above marginal cost, then so are the equilibrium personalized prices, meaning that firms can achieve positive expected profits on the segments on which they profile consumers. To state the result, we define $p_{B}^{x} \equiv \min \left\{p_{B}, r(x)\right\}$ as the maximum personalized price that firm $A$ can set to attract consumer $x$ when firm $B$ does not profile this consumer; as the latter event occurs with conditional probability $1-b$, firm $A$ can secure an expected profit of $(1-b) p_{B}^{x}$ for any consumer 
belonging to the $A$-translucent segment. We define $p_{A}^{x} \equiv \min \left\{p_{A}, r(x)\right\}$ accordingly; then, the expected profit that firm $B$ can secure from consumers on the $B$-translucent segment is equal to $(1-a) p_{A}^{x}$. In what follows, we assume (without loss of generality) that $(1-b) p_{B}^{x} \geq(1-a) p_{A}^{x} \sqrt{15}$ The next proposition describes the second-stage equilibrium when at least one firststage uniform price is strictly above marginal cost (the proof with the full characterization can be found in Appendix 7.1).

Proposition 1. Given uniform prices $p_{A}$ and $p_{B}$, and $r(x)$, and assuming that $(1-b) p_{B}^{x}>0$, then

- If $(1-b) p_{B}^{x}>(1-a) p_{A}^{x}$, there exists a unique second-stage personalized pricing equilibrium with the following features:

1. If $p_{A}^{x} / p_{B}^{x} \leq a(1-b)$, the equilibrium is in mixed strategies, firm $A$ has an expected payoff of $(1-b) p_{B}^{x}$ and firm $B$ has an expected payoff of $a(1-b) p_{B}^{x}$;

2. If $a(1-b)<p_{A}^{x} / p_{B}^{x}<1-b$, the equilibrium is in mixed strategies if $p_{A}^{x} / p_{B}^{x}<a$ or in pure strategies otherwise; in any case, firm $A$ has an expected payoff of $(1-b) p_{B}^{x}$ and firm $B$ has an expected payoff of $p_{A}^{x}$;

3. If $p_{A}^{x} / p_{B}^{x} \geq 1-b$, the equilibrium is in mixed strategies and both firms have an expected payoff of $(1-b) p_{B}^{x}$.

- If $(1-b) p_{B}^{x}=(1-a) p_{A}^{x}$ there exists a mixed strategy equilibrium in which both firms have an expected payoff of $(1-b) p_{B}^{x}=(1-a) p_{A}^{x}$.

At first glance, the price equilibrium in mixed strategies described in Proposition 1 could be seen as a replay of the logic proposed by Varian (1980), with captive consumers (who only know the price of one firm) and shoppers (who know both prices). Yet, the driver of price randomization in our setting is not consumers' information about pricing, but firms' information about the consumer segments to which their prices are being targeted; that is, when one firm targets a customer, it does not know whether it has unique knowledge of the customer's valuation or whether there is competition from the other firm.

\footnotetext{
${ }^{15}$ The construction of the unique Nash equilibrium when $(1-a) p_{A}^{x}<(1-b) p_{B}^{x}$, as detailed in Appendix 7.1, does not depend on the assumption that $a>b$. In other words, the equilibrium we construct does not depend on the identity of the firm $A$ or $B$ : If $(1-a) p_{A}^{x}>(1-b) p_{B}^{x}$, we obtain an equivalent proposition by interchanging $p_{A}$ and $p_{B}$, as well as the parameters $a$ and $b$ in Proposition 1
} 


\subsection{Uniform pricing equilibrium}

While Lemma 1 states that marginal cost pricing can be a Nash equilibrium of the two stage pricing game, Proposition 1 suggests that it cannot be a subgame-perfect Nash equilibrium. The reason is the following: firms can expect strictly positive profits at the second stage of the game as soon as at least one uniform price is strictly positive; hence, a forward-looking firm cannot find it optimal to set its uniform price equal to marginal cost. There are, however, two extreme cases in which this reasoning breaks down, as stated in the following lemma.

Lemma 2. Marginal cost pricing, i.e., $\left.\left(p_{A}, p_{B}\right)=p_{A}(x), p_{B}(x)\right)=(0,0)$, is the unique SPNE of the game in either of the following two cases: (1) $\beta=0$, or (2) $\beta=1$ and $\phi=1 / \lambda$.

Proof. Proof. In case (1), as firm $B$ is not able to identify any consumer, only the opaque and the $A$-translucent segments exist. Suppose by contradiction that firm $B$ sets $p_{B}>0$. Then, firm $B$ does not make any sales on the $A$-translucent segment because firm $A$ can always undercut any offer $p_{B}>0$ with a personalized price. It follows that firm $B$ competes only in the opaque segment, on which Bertrand competition ensues: for any $p_{B}>0$, firm $A$ has an incentive to slightly undercut this price, which yields to a unique equilibrium with $\left(p_{A}, p_{B}\right)=(0,0)$ and, by Lemma 1. $\left(p_{A}(x), p_{B}(x)\right)=(0,0)$. In case (2) ${ }^{16}$ firms are symmetric: they identify exactly the same consumers as their profiling technologies are perfectly correlated and equivalent. Because the two translucent segments are empty, all strategic links between the opaque and transparent segments are severed. On the transparent segment, 'classic' Bertrand competition prevails for each and every consumer. It follows that equilibrium personalized prices are $\left(p_{A}(x), p_{B}(x)\right)=(0,0)$ for all $x$. This equilibrium outcome is independent of any uniform price $p_{K}$ that may prevail on the opaque segment. As a consequence, competition for consumers on the opaque segment is also equivalent to Bertrand competition, leading to uniform prices that also equal marginal cost in equilibrium: $\left(p_{A}, p_{B}\right)=(0,0)$.

We now show that outside the two extreme cases of Lemma 2, firms have market power at the SPNE of the game. As shown in Proposition 1, the expected payoff for firm $A$ in the second-stage equilibrium is an increasing function of $p_{B}^{x}$. As a consequence, firm $B$ faces a trade-off when raising its uniform price in the first stage of the game: on the one hand, a higher $p_{B}$

\footnotetext{
${ }^{16}$ Technically, Proposition 1 does not apply to this case: as $b=\phi \beta \lambda=1$, it follows that $(1-b) p_{B}^{x}=0$, which violates one of the assumptions on which Proposition 1 relies.
} 
increases expected profits on the transparent and $B$-translucent segments (as it induces firm $A$ to increase its prices), but on the other hand, it lowers expected profits on the opaque and $A$-translucent segment (as it makes it easier for firm $A$ to undercut $B$ 's price).

Given this trade-off, one option for firm $B$ is to focus exclusively on the segments where it profiles consumers and to forgo any profit on the segments where it does not profile any consumer. To do so, firm $B$ simply needs to set $p_{B}>p_{A}$ for any $p_{A}$. What would be firm $B$ 's expected profit be under that option? From Proposition 1, we know that the payoff firm $B$ expects from any consumer $x$ is weakly increasing in $p_{B}$. It follows that if firm $B$ chooses to focus only on consumers that it happens to profile, it will set the largest possible uniform price: $p_{B}=\bar{r}$. We can then compute the profit that firm $B$ can expect for some $p_{A}$ set by firm $A$. Recalling that $p_{B}^{x} \equiv \min \left\{p_{B}, r(x)\right\}$, we have here that $p_{B}^{x}=r(x)$. Now, applying Proposition 1, we know that firm $B$ 's profit is equal to: ${ }^{17}$

1. $(1-b) r(x)$ for $p_{A}^{x} / p_{B}^{x} \geq 1-b$, or $r(x) \leq p_{A} /(1-b)$,

2. $p_{A}$ for $a(1-b)<p_{A}^{x} / p_{B}^{x}<1-b$ or $p_{A} /(1-b)<r(x)<p_{A} /(a(1-b))$, and

3. $a(1-b) r(x)$ for $p_{A}^{x} / p_{B}^{x} \leq a(1-b)$ or $r(x) \geq p_{A} /(a(1-b))$.

Aggregating over all consumers, we thus have:

$\stackrel{\circ}{\pi}_{B}\left(\bar{r}, p_{A}\right)=\int_{0}^{\frac{p_{A}}{1-b}}(1-b) r f(r) d r+\int_{\frac{p_{A}}{1-b}}^{\frac{p_{A}}{a(1-b)}} p_{A} f(r) d r+\int_{\frac{p_{A}}{a(1-b)}}^{\bar{r}} a(1-b) r f(r) d r$.

As the total mass of the transparent and $B$-translucent segments is $M_{2}+$ $M_{B}=\lambda_{B}=\beta \lambda$, this strategy will guarantee firm $B$, for some $p_{A}$ set by firm $A$, an expected profit of

$$
\pi_{B}^{\min }\left(p_{A}\right)=\beta \lambda \stackrel{\circ}{\pi}_{B}\left(\bar{r}, p_{A}\right)
$$

If firm $B$ chooses $p_{B}=\bar{r}$, then firm $A$ 's best response is to set the monopoly price, $p^{m}$, which solves $\max _{p} p S(p){ }^{18}$ This is so for two reasons. First, firm $A$ is the only active firm on the opaque and $A$-translucent segments since firm $B$ priced itself out of these segments. Second, the uniform price that firm $A$ 's chooses bears no impact on the expected profit that it obtains afterwards when setting its personalized prices (see Proposition 1).

\footnotetext{
${ }^{17}$ It is easily checked that $p_{B}=\bar{r}$ implies that $(1-b) p_{B}^{x} \geq(1-a) p_{A}^{x}$.

${ }^{18}$ Our assumption that demand is log-concave makes sure that such price exists.
} 
We need now to examine under which condition setting $p_{B}=\bar{r}$ is firm $B$ 's best response when firm $A$ sets $p_{A}=p^{m}$. The best alternative for firm $B$ is to set $p_{B}=p^{m}-\varepsilon$, so as to compete as well on the opaque and $A$ translucent segments without sacrificing too much profit on the transparent and $B$-translucent segments later on. What would be firm $B$ 's expected profit in that case? With probability $M_{2}+M_{B}$, firm $B$ competes for consumers on the transparent and $B$-translucent segments, on which it obtains expected profits (almost) equal to

$$
\stackrel{\circ}{\pi}_{B}\left(p^{m}, p^{m}\right)=(1-\beta \phi \lambda)\left[\int_{0}^{p^{m}} r f(r) d r+\int_{p^{m}}^{\bar{r}} p^{m} f(r) d r\right] .
$$

With probability $M_{0}$, it competes on the opaque segment and, as it slightly undercuts firm $A$, it achieves profits (almost) equal to $\pi^{m} \equiv p^{m} S\left(p^{m}\right)$. Finally, with probability $M_{A}$, it competes on the $A$-translucent segment, where it makes (almost certainly) no sales and obtains (almost) zero profit. In sum, firm $B$ 's best expected deviation profit when firm $A$ sets its uniform price equal to the monopoly price is computed as

$$
\left.\Pi_{B}^{e}\left(p^{m}\right)\right|_{p_{A}=p^{m}}=\beta \lambda \stackrel{\circ}{\pi}_{B}\left(p^{m}, p^{m}\right)+[1-\lambda-\beta \lambda(1-\phi \lambda)] \pi^{m} .
$$

Hence, if $\pi_{B}^{\min }\left(p^{m}\right) \geq\left.\Pi_{B}^{e}\left(p^{m}\right)\right|_{p_{A}=p^{m}}$, there exists a pure-strategy equilibrium in which firm $A$ is a monopolist in the segments where consumers are not profiled or only by firm $A$, while firm $B$ gives up any sales to consumers it does not profile and chooses a uniform price that maximizes its expected profits in the transparent segment. Using expression (2) and (3), we find that $\pi_{B}^{\min }\left(p^{m}\right) \geq\left.\Pi_{B}^{e}\left(p^{m}\right)\right|_{p_{A}=p^{m}}$ is equivalent to:

$$
\beta \lambda\left[\stackrel{\circ}{\pi}_{B}\left(\bar{r}, p^{m}\right)-\stackrel{\circ}{\pi}_{B}\left(p^{m}, p^{m}\right)\right] \geq(1-\lambda-\beta \lambda(1-\phi \lambda)) \pi^{m} .
$$

Otherwise, if Condition (4) is not met, firm $B$ will choose to compete as well for the consumers that it does not profile. But then, firm $A$ will have no reason to set the monopoly price. As competition ensues on the opaque segment, both firms must randomize uniform prices, as it is the only way for them to make positive profits and, thereby, to be indifferent with the previous situation where firm $B$ stays out of the opaque and $A$-translucent segments (and firm $A$ monopolizes them).

We have thus proven the following proposition.

Proposition 2. In the first stage of the game, the pair $\left(p_{A}, p_{B}\right)=\left(p^{m}, \bar{r}\right)$ is an equilibrium in pure strategies if and only if $\pi_{B}^{\min }\left(p^{m}\right) \geq\left.\Pi_{B}^{e}\left(p^{m}\right)\right|_{p_{A}=p^{m}}$, which is equivalent to Condition (4). Otherwise, the equilibrium is in mixed strategies. 
We now draw a number of insightful implications for data markets and for consumers. Where needed, we will base our analysis on the specific case of perfectly correlated profiling technologies, for which we are able to fully characterize the subgame-perfect Nash equilibrium.

\section{Implications for data markets}

So far, we have not been very specific regarding the origin of the profiling technologies. We have simply indicated that profiling was made possible by the use of data and customer analytics; we also stated that it was reasonable to assume that the two profiling technologies were correlated if firms had access to 'similar' data sets. Two scenarios fit the latter assumption quite well: either one of the firms collected data and, then, decided to share the customer analytics with the other firm; or customer analytics was produced by third parties (so-called 'data brokers') and then sold to the firms. In either scenario, the data providers' strategies on the supply side are shaped by the data users' willingness to pay on the demand side. Our previous results are thus instrumental to shed light on the working of data markets, as they tell us about the profits that competing firms can extract from their use of data and customer analytics for differential pricing purposes.

A first important finding can be drawn from Lemma 2, which shows that equilibrium profits are nil if either only one firm is able to profile consumers (i.e., if $\beta=0$ ), or both firms can profile the exact same set of consumers because their profiling technologies are symmetric and perfectly correlated (i.e., if $\beta=1$ and $\phi=1 / \lambda$ ). The immediate consequence is that any (for-profit) data supplier will chose to provide both firms with differentiated customer analytics. The fact that both firms should be provided with data implies, in the first scenario, that a firm that collects data should share it with its competitor; in the second scenario, it means that data brokers should avoid exclusive data contracts, the opposite recommendation to the one drawn from previous studies (see Braulin and Valletti (2016), and Montes et al. (2019)).

Regarding the necessary differentiation of the customer analytics, what our model tells us is that data suppliers must make sure that the competing firms end up profiling different sets of consumers. Analyzing the optimal way to achieve this goes beyond the scope of this paper ${ }^{19}$ However, we can give useful indications as to how the asymmetry $(\beta)$ and correlation $(\phi)$ of the profiling technologies should be calibrated. As shown in Proposition 2 ,

\footnotetext{
${ }^{19}$ We cannot, in our limited setting, consider the full set of strategies that data suppliers can choose from. For instance, they can divide customer data in overlapping or nonoverlapping tranches.
} 
the first stage of the game has a pure-strategy equilibrium if Condition (4) is met. In this equilibrium, firm $B$ chooses to maximize its profits on the segments where it profiles consumers, while letting firm $A$ monopolize the other segments. As total (expected) profits are maximized in this equilibrium, it is in the interest of any data supplier to design its offering so that this equilibrium is more likely to occur ${ }^{20}$ To see how, we rewrite Condition (4) as follows:

$$
\begin{array}{r}
\int_{p^{m}}^{\frac{p^{m}}{1-\beta \phi \lambda}} \beta \lambda(1-\beta \phi \lambda)\left(r-p^{m}\right) f(r) d r+\int_{\frac{p^{m}}{1-\beta \phi \lambda}}^{\frac{p^{m}}{\phi(1-\beta \phi)}} \beta^{2} \lambda^{2} \phi p^{m} f(r) d r \\
+\int_{\frac{p^{m}}{\phi \lambda(1-\beta \phi \lambda)}}^{\bar{r}} \beta \lambda(1-\beta \phi \lambda)\left(\phi \lambda r-p^{m}\right) f(r) d r \\
\geq(1-\lambda-\beta \lambda(1-\phi \lambda)) \pi^{m} .
\end{array}
$$

In the case of perfectly correlated profiling technologies $(\phi=1 / \lambda)$, Condition (5) boils down to

$\int_{p^{m}}^{\frac{p^{m}}{1-\beta}} \beta \lambda(1-\beta)\left(r-p^{m}\right) f(r) d r+\int_{\frac{p^{m}}{1-\beta}}^{\bar{r}} \beta \lambda(1-\beta)\left(r-p^{m}\right) f(r) d r \geq(1-\lambda) \pi^{m}$,

which can be rewritten as

$$
\beta(1-\beta) \geq \frac{1-\lambda}{\lambda} \frac{\pi^{m}}{C S^{m}}
$$

where $C S^{m} \equiv \int_{p^{m}}^{\bar{r}}\left(r-p^{m}\right) f(r) d r$ is the consumer surplus under (uniform) monopoly pricing. As the right-hand side is independent of $\beta$, it is easily seen that the latter condition is most likely to be satisfied when $\beta=1 / 2$. Hence, if data providers were to provide firms with perfectly correlated profiling technologies, they would set the level of asymmetry between the technologies at $\beta=1 / 2$, so as to extract the largest rents from the firms. To understand this result, note that an increase in $\beta$ expands the set of customers that firm $B$ is able to profile, and so (other things being equal), linearly increases demand. However, we show in Appendix 7.2 (where we develop the perfect correlation case) that the lower bound to the price charged (and so the expected payoff per customer) is proportional to $1-\beta$. It follows that firm $B$ 's profits from profiled customers are proportional to $\beta(1-\beta)$. So, one way to interpret the previous result is to say that the higher the uncertainty about the information

\footnotetext{
${ }^{20}$ To see this, note that expected payoff from profiling for both firms (which happens with joint probability mass $M_{2}+M_{A}+M_{B}$ ), is maximized when $p_{B}=\bar{r}$. In addition, the payoff on the opaque segment (which occurs with probability $1-M_{2}+M_{A}+M_{B}$ ) is maximized when $\min \left\{p_{A}, p_{B}\right\}=p^{m}$. So, in any of the events (when there is profiling and when there is none), the sum of expected profits of both firms is maximized.
} 
firm $B$ has, the higher the prices (as it is precisely this uncertainty that drives the randomization of prices); in the case of perfectly correlated technologies, the maximum uncertainty is reached at $\beta=1 / 2{ }^{21}$

As expression (5) is continuous in $\phi$ on the interval $\left[1, \frac{1}{\lambda}\right]$, the conclusion that it is optimal to have some level of asymmetry holds more generally for a range of imperfectly correlated profiling technologies. Interestingly, we can say a lot more in the case of a relatively high monopoly price, namely $p^{m} \geq(1-\beta \phi \lambda) \bar{r}$. Condition (5) simplifies then to

$$
\int_{p^{m}}^{\bar{r}} \beta \lambda(1-\beta \phi \lambda)\left(r-p^{m}\right) f(r) d r \geq(1-\lambda-\beta \lambda(1-\phi \lambda)) \pi^{m},
$$

which is equivalent to

$$
L(\beta) \equiv \beta \lambda(1-\phi \beta \lambda)-(1-\lambda-\beta \lambda(1-\phi \lambda)) R^{m} \geq 0,
$$

where $R^{m}$ stands for $\pi^{m} / C S^{m}$.

We find that

$$
\frac{\partial L}{\partial \beta}=0 \Leftrightarrow \beta=\frac{(1-\lambda \phi) R^{m}+1}{2 \lambda \phi} \equiv \beta^{*},
$$

with $\beta^{*}=1 / 2$ for $\phi=1 / \lambda$ and $\beta^{*}>1 / 2$ for $\phi<1 / \lambda$. We see that it is not optimal for data providers to propose symmetric profiling technologies when profiling technologies are sufficiently correlated (which generalizes our previous result); formally,

$$
\beta^{*}<1 \Leftrightarrow \phi>\frac{R^{m}+1}{R^{m}+2} \frac{1}{\lambda} .
$$

To sum up, our analysis of the differential pricing game gives us precious information about the profits that firms can expect for a given pair of profiling technologies. From there, we can evaluate the firms' willingnesses to pay for various offerings of customer analytics and so, the revenues that data providers can achieve by providing these offerings. If the data provider is one of the two firms, we show that it has an incentive to share its means to profile consumers with its rival, but not entirely, so that the data provider remains 'better informed' than its rival. This can be achieved by selling to the rival a profiling technology that is imperfectly correlated and/or asymmetric with the data provider's technology. Intuitively, when the less-informed firm

\footnotetext{
${ }^{21}$ Interestingly, Ireland (1993) reaches a similar result in a different context. In his twostage Varian-like model, firms choose first the fraction of consumers who will be made aware of their existence and next, the uniform price at which to sell their good.
} 
has a very poor profiling ability compared to the better-informed firm, it mainly competes in the opaque segment. So, improving the less-informed firm's ability enables it to also compete in the transparent segment, which in turn relaxes competition in the opaque segment. However, when the profiling abilities of the two firms are very similar, the two firms mainly compete in the transparent segment. Improving the less-informed firm's ability then makes competition in the transparent segment even more intense, which in turn leads to more competition in the opaque segment. The same intuition applies when profiling technologies are sold by third-parties. A monopoly data broker will follow the same logic and target the two firms with differentiated offerings. Similarly, two data brokers who cannot offer themselves a menu of options will vertically differentiate the offerings they sell at equilibrium 2

\section{Implications for consumers}

We investigate now how competitive differential pricing affects the surplus of consumers. As the two firms produce a homogeneous product, we know that in the absence of differential pricing, the unique Nash equilibrium is such that prices are equal to marginal cost; consumer surplus reaches then its maximum. We also know from Lemma 2 that the possibility to price discriminate is not a sufficient condition for departing from this benchmark: if only one firm can profile consumers or if both firms can profile the exact same set of consumers, marginal cost pricing continues to prevail at equilibrium.

This means that for consumers to be hurt, both firms must profile consumers but with different abilities. Yet, as we explained in the previous section, this situation should be the norm rather than the exception, as it serves the interests of data providers. It is thus legitimate to fear the negative impacts that the data revolution may have on consumer welfare, as eloquently expressed by Newman (2013):

"The darker version of online marketing is that it can facilitate what economists call 'price discrimination,' selling the same exact good at a variety of prices in ways unknown to the buyers. This is based on the reality that people have different maximum prices that they are willing to pay, a so-called 'pain point' after which they won't buy the

\footnotetext{
${ }^{22}$ In that case, the data broker who sells the offering leading to the best profiling ability will earn more profits (ignoring investments costs). In Appendix 7.2, we show indeed that when profiling technologies are perfectly correlated, the better-informed firm achieves higher expected profits than the less-informed firm. By continuity, this result also applies to highly but imperfectly correlated technologies.
} 
product. The ideal for a seller would be to sell a product to each customer at their individual 'pain point' price without them knowing that any other deal is available."

Such fears have led the Council of Economic Advisers of the Whitehouse to release a study on big data and differential pricing in 2015, in which it is equally recognized that firms may be engaging in random price testing ${ }^{23}$

How does our analysis contribute to this debate? Although it seems extremely hard to perform a general analysis of the impacts of competitive differential pricing on consumer surplus ${ }^{24}$ we are able to shed some light on a number of interesting issues when we focus on the specific case of perfectly correlated profiling technologies.

In a nutshell, we show that consumers are unequal in front of improved profiling: although many consumers may lose (and would thus be willing to protect their privacy), some other consumers may win; it may even be the case that the winners would benefit sufficiently so that they could compensate the losers, i.e., consumers as a group would welcome improved profiling.

In the rest of this section, we focus on the case perfectly correlated profiling technologies, i.e., we assume that $\phi=1 / \lambda$, so that $a=1$ and $b=\beta$. We also assume that firm $B$ is able to profile consumers $(\beta>0)$ but not as well as firm $A$ ( $\beta<1$, profiling technologies are asymmetric), so that Lemma 2 does not apply. Under these assumptions, any consumer that firm $B$ (the 'lessinformed' firm) profiles is also profiled by firm $A$ (the 'better-informed' firm), meaning that the $B$-translucent segment is empty $\left(M_{B}=\beta \lambda(1-\phi \lambda)=0\right)$. This greatly simplifies the analysis and allows us to fully characterize the subgame-perfect Nash equilibrium, perform comparative statics exercises, and draw insightful results about the sensitivity of consumer surplus and the demand for privacy.

\footnotetext{
${ }^{23}$ See Executive Office of the President of the United States (2015).

${ }^{24}$ Writing down the consumer surplus supposes being able to complete the following list of tasks. First, for each consumer with value $r$, we need to obtain the (expected) prices that she is likely to face, taking into account that she will face (i) the minimum of the two (potentially random) uniform prices if she is not profiled by any firm, (ii) the minimum of both firms' random personalized prices if she is profiled by both firms, or (iii) the minimum of firm $A$ 's random personalized price and firm $B$ 's (potentially random) uniform price if she is profiled only by firm $B$ (and inversely if she is only profiled by firm $B$ ). Second, for each minimum of prices, we need to calculate the densities of these minimum prices and the corresponding surplus to this consumer. Third, we need to integrate over all prices to obtain the expected surplus of this consumer. Finally, we need to integrate over all consumers to get the total consumer surplus. One understands that these operations are complex to perform, even in the case of simple distributions such as the uniform distribution. Moreover, performing comparative static exercises would add another layer of complexity.
} 


\subsection{Equilibrium characterization and comparative stat- ics}

Developing Propositions 1 and 2 for the case under review, we obtain the following characterization of the subgame-perfect equilibrium (the proof and the exact formulation of some functions are relegated to Appendix 7.2).

Proposition 3. If profiling technologies are perfectly correlated, there exists a subgame-perfect Nash equilibrium such that: (1) At the first stage, firms choose their uniform price according to the following strategies. Firm A draws $p_{A}$ from the distribution $H_{A}(p)$ with probability $\nabla_{A}$, or sets $p_{A}=p^{m}$ with probability $1-\nabla_{A}$. Firm $B$ draws $p_{B}$ from the distribution $H_{B}(p)$ with probability $\nabla_{B}$, or sets $p_{B}=\bar{r}$ with probability $1-\nabla_{B}$. The distributions $H_{A}(p)$ and $H_{B}(p)$ are defined on the interval $\left[p_{l}, p^{m}\right)$. If Condition (6) is met, then $\nabla_{A}=\nabla_{B}=0$ and the pair $\left(p_{A}, p_{B}\right)=\left(p^{m}, \bar{r}\right)$ is an equilibrium in pure strategies.(2) At the second stage, if $p_{B}=0$, then $\left(p_{A}(x), p_{B}(x)\right)=$ $(0,0)$ is the unique equilibrium; if $p_{B}>0$, firm $B$ draws $p_{B}(x)$ from the distribution $G^{x}(p)$, with

$$
G^{x}(p)= \begin{cases}\frac{p-(1-\beta) p_{B}^{x}}{\beta p} & \text { for } p \in\left[(1-\beta) p_{B}^{x}, p_{B}^{x}\right], \\ 0 & \text { otherwise }\end{cases}
$$

and $p_{B}^{x} \equiv \min \left\{p_{B}, r(x)\right\}$; firm $A$ does so as well with probability $\beta$, and sets $p_{A}(x)=p_{B}^{x}$ with probability $1-\beta$.

Our objective is now to understand how the equilibrium prices depend, for any $\log$ concave demand function, on two key parameters of the model: the measure of firms' abilities to profile consumers $(\lambda)$ and the level of asymmetry between the two profiling technologies $(\beta)$.

Improved profiling. How do firms modify their pricing behavior when their ability to profile consumers improves, i.e., when $\lambda$ increases? Recall that we assume that the profiling technologies of the two firms are perfectly correlated, with respective abilities to profile consumers $\lambda_{A}=\lambda$ and $\lambda_{B}=\beta \lambda$. Hence, an increase in $\lambda$ makes it easier for both firms to profile consumers. The following corollary summarizes the answer to this question. ${ }^{25}$

Corollary 1. An improved ability to profile consumers increases the uniform prices in the sense of first-order stochastic dominance, and it does not affect the distribution of personalized prices. Hence, an increase in $\lambda$ leads to higher uniform and personalized prices.

\footnotetext{
${ }^{25}$ The proofs of all comparative statics results can be found in Appendix 7.2 .2
} 
It is clear from Proposition 3 that for a given uniform price of firm $\mathrm{B}, p_{B}$, the distribution of personalized prices is independent of $\lambda$. However, increasing $\lambda$ has three effects on the uniform prices. First, it increases the lowest possible uniform price charged by both firm $A$ and firm $B$, i.e. $\partial p_{l} / \partial \lambda>0$. Second, it increases the probability that both firms charge their respective highest uniform price, i.e. $\partial \nabla_{A} / \partial \lambda<0$ and $\partial \nabla_{B} / \partial \lambda<0$. Finally, they tend to charge higher prices when they randomize their uniform prices, i.e. $\partial H_{A}(p) / \partial \lambda<0$ and $\partial H_{B}(p) / \partial \lambda<0$. In sum, all three effects push the distributions of uniform prices to the right. Since $\lambda$ has no effect on the distribution of equilibrium personalized prices, we can be sure that an improved ability to profile consumers leads, ceteris paribus, to higher prices. Intuitively, when profiling is improved, it is more likely that firms will be competing in the transparent segment, which relaxes competition in the opaque segment and thus raises uniform prices.

More symmetric profiling technologies. Let us now consider the effect on the pricing behavior of both firms of increasing the symmetry between the profiling technologies (measured by the parameter $\beta$ ). It would seem logical to expect that as the profiling technologies become more similar, competition between the two firms would get more intense and prices would go down. However, things are not as simple here, as stated in the following corollary.

Corollary 2. The uniform prices are at their highest level in the sense of first-order stochastic dominance when the level of asymmetry is intermediate (i.e., $\beta=1 / 2$ ).

As above, we can show that the lower bound of the uniform price distribution, the probability of firms charging the highest uniform price, and the probability of firms charging higher uniform prices when they randomize their uniform prices are at their highest level when $\beta=1 / 2$. This implies that more symmetry increases the uniform prices when firms' detection technologies are very different, but decreases the uniform prices when firms' technologies are slightly different. There is thus an intermediate level of asymmetry, $\beta=1 / 2$, that maximizes the profits of the two firms.

Another effect of increasing $\beta$ is to push personalized prices down for given uniform prices. This is due to the more intense competition between firms on the transparent segment ${ }^{26}$

Corollary 3. For given uniform prices, the personalized prices decrease with the symmetry between firms in the sense of first-order stochastic dominance.

\footnotetext{
${ }^{26}$ However, this result ignores the indirect effect a higher level of symmetry has on personalized prices through the effect it has on uniform prices. The expected uniform
} 
In Appendix 7.2, we illustrate the previous comparative statics results with the case of a uniform distribution of the consumer valuations.

\subsection{Do consumers necessarily suffer from improved pro- filing?}

To answer this question formally, we decompose the effects that improved profiling may have on different categories of consumers. We know from Corollary 1 that an increase in $\lambda$ decreases the probability that either firm will charge any uniform price below some price $p$. This unequivocally implies that all prices go up. It may then seem natural to conclude that the consumer surplus goes down. But there is a twist: the impact of these higher prices is only clear for consumers whose status does not change, i.e., those who remain anonymous (who face higher uniform prices) and those who remain profiled (who face higher personalized prices). Yet, the impact on consumers who switch status remains unclear. Improved profiling indeed means that some consumers who used to be anonymous are now profiled. Among them, consumers with a low valuation will start purchasing (which they did not do when they were anonymous), and will thus enjoy a larger surplus; consumers with a larger valuation, who continue to purchase, may also benefit if they are now charged a lower personalized price than the uniform price they were paying before. As the following example shows, the positive impact on 'switchers' may be sufficiently important to outweigh the negative impact on 'non switchers', so that improved profiling ends up increasing consumer surplus on aggregate.

In Appendix 7.2, we compute the consumer surplus in the case of a uniform distribution of consumer valuations; we show that it is an increasing function of $\lambda$ in this particular case. To understand this result, let us divide the group of 'switchers' into three subgroups. We have first the 'Low value switchers' (group L), who did not purchase before precision improved; then we have the 'Middle value switchers' (group $M$ ), who would have purchased at the monopoly price but have now access, with higher precision, to a lower price (in expectation); finally, we have the 'High value switchers' (group $H$ ), who would have purchased at the monopoly price but are now faced with a

price is:

$$
E p_{B}=\left(1-\nabla_{B}\right) \bar{r}+\nabla_{B} \int_{p_{l}}^{p^{m}} p h_{B}(p) d p=\left(1-\nabla_{B}\right) \bar{r}+\nabla_{B}\left[p_{l}+\int_{p_{l}}^{p^{m}}\left(1-H_{B}(p)\right) d p\right] .
$$

We can show that $\partial E p_{B} / \partial \beta>0$ when $\beta<1 / 2$, which means that the expected highest personalized prices are maximized when $\beta=1 / 2$. 
targeted price that is higher than the monopoly price in expectations (these consumers would prefer to 'hide'). In the present example, the benefits to groups $L$ and $M$ (a market expansion effect) outweigh the losses for group $H$ (a price discrimination effect). Moreover, the benefits for switchers with low and middle valuations also outweigh the losses for all consumers who do not switch status.

But if consumers as a group benefit, does it necessarily imply that firms will have lower profits? The answers is no. In fact, in the above example (see Appendix 7.2 for the details), firm $A$ 's profit remain constant, while firm $B$ 's profit increases when $\lambda$ increases. Hence, the market expansion effect dominates for firms as well. This is no surprise for firm $B$, since it only obtains positive profits on the transparent segment and prices in the transparent segment are not affected by an increase in $\lambda$. For firm $A$ the loss of profits on the opaque segment is just compensated by the gain in profits in the transparent and translucent segments. Hence, somewhat surprisingly, while consumers as a group are better off on average, this does not happen at the expense of the firms' profits. To the contrary, consumers (as a group) and firms (as a group) strictly benefit from higher precision. This is due to the market expansion effect and the corresponding reduction in the deadweight loss.

We can also study how consumers are affected when only the profiling technology of the less-informed firm improves (i.e., when $\beta$ increases). In this case, we see from Corollary 3 that the impact depends of where we start from. For relatively high levels of symmetry between firms' profiling technologies $(\beta>1 / 2)$, increasing further the symmetry benefits consumers as it leads to both lower uniform prices and lower personalized prices. In contrast, for relatively low levels of symmetry $(\beta<1 / 2)$, increasing the symmetry leads to higher uniform prices but lower personalized prices.

\subsection{Exogenous and endogenous privacy}

So far, we have assumed that it is not possible for consumers to escape being identified by the firms when profiling technologies are effective. In reality, consumers may resort to obfuscation strategies that make profiling technologies inoperative; for instance, consumers may delete cookies, use tools to browse the web anonymously, or purchase ad blockers. Although a full analysis of this possibility goes beyond the scope of this paper, we can discuss under which conditions consumers may wish to 'hide' themselves. ${ }^{27}$

\footnotetext{
${ }^{27}$ To analyze the full implications of endogenous privacy choices by consumers, we would first need to assess the interplay between consumers' hiding behavior and firms' pricing
} 
As we explained in Section 3, firm B may, when the opaque segment is relatively small, have an incentive to focus only on consumers it can profile, by charging the choking price of demand as its uniform price, while firm A charges the monopoly price as its uniform price. This pushes up prices on the transparent segment, leading in some cases to a situation in which all personalized prices lie above the monopoly price (charged to consumers that a firm does not profile). In this case, some of these consumers would prefer to hide, as they will face lower prices in the opaque segment. We saw in Corollary 1 that this situation becomes more likely the higher the precision of the detection technology. This implies that the less privacy regulation there is (the higher $\lambda$ ), the more it will lead to a demand for (endogenous) privacy. As a consequence, better privacy regulation (lower $\lambda$ ) would tend to push prices down, increasing consumer surplus, and lower the need for consumers to hide. We conclude that the fear for consumers to face higher prices when they are 'in the open' rather than 'in the dark' does not disappear when it is not just one but several competing firms that have the ability to profile consumers.

\subsection{What if uniform prices are made public?}

We now study the consequences of having 'observable' uniform prices. We have in mind what is commonly known as list prices, i.e., (uniform) prices that any consumer can observe and can thus claim to buy at. The immediate consequence of the presence of such prices is that firms now face an upper bound on the personalized prices they can charge to the consumers they profile. Hence, a firm does not just compete against the prices of its competitor but also against its own uniform price. We expect that this would lead to more competition and lower prices in equilibrium. We provide two results that allow us to confirm this conjecture.

First, it is immediate that setting all prices equal to marginal cost is now a subgame-perfect equilibrium of the game. In contrast to the case where consumers only receive one price from each firm, no firm has an incentive to increase its uniform price at stage 1, as all consumers (profiled or not) always keep the possibility to purchase at the lowest listed uniform price. We conclude that the observability of uniform prices restores the Bertrand Paradox even when firms have asymmetric profiling technologies.

strategies; we could then characterize an equilibrium with endogenous hiding, and study the effect of consumer hiding on firms' profits and consumer surplus. We leave this for future research. 
Proposition 4. If all consumers can purchase a good at the (observed) uniform price, then marginal cost pricing is a subgame-perfect Nash equilibrium of the pricing game.

What remains to be shown, however, is whether this is the only equilibrium. Our second result gives an indicative answer. We show in the following example that, in a simplified version of our model where consumers can have valuations of only two types, high and low, no other equilibrium can exist.

\section{Example: A model with two types of consumers.}

Assume that there is a unit mass of consumers and that any consumer $x$ can be one of two types: their valuation for the homogeneous product is either high, $r(x)=1$, with probability $\varphi \in(0,1)$ or low, $r(x)=\zeta<1$, with probability $1-\varphi$. Assume further that if the market was served by a (non discriminating) monopolist, this firm would set a uniform price equal to $\zeta$ and so, serve the whole market; this happens when $\varphi<\zeta$. In this setting, we can show (see Appendix 7.3) that there cannot exist a subgame-perfect Nash equilibrium of the game with the features described in Proposition 3 (i.e., both firms draw from a connected interval $[\underline{p}, \zeta)$ in which firm $A$ draws $p_{A}$ according to the distribution $H_{A}(p)$ with probability $\nabla_{A}$, or sets $p_{A}=\zeta$ with probability $1-\nabla_{A}$, and in which firm $B$ draws $p_{B}$ from the distribution $H_{B}(p)$ with probability $\nabla_{B}$, or sets $p_{B}=\zeta$ with probability $\left.1-\nabla_{B}\right)$.

Given the previous two findings, there are good reasons to believe that list prices exert downward pressure on equilibrium prices in our model.

\section{Discussion and concluding remarks}

We have examined price competition in a homogenous goods setting in which firms can imperfectly profile consumers. When they do, they can charge these consumers a personalized price, but firms (potentially) differ in their ability to profile. This asymmetry between the firms' profiling technologies leads to uncertainty about the nature of price competition and, thereby, generates market power. The necessary ingredients for this result are price competition and 'imperfect consumer profiling', which refers to the idea that firms are able to identify consumers' valuations for the product but only in an imperfect way. The profiling of consumers enables price discrimination, but its imperfect nature makes firms uncertain about the competitor's pricing strategy, which triggers strategic random pricing. In sum, as long as both 
firms can track consumers, asymmetric and/or imperfectly correlated profiling technologies can generate a way out of the Bertrand paradox. Our results also imply that when only one firm can profile consumers, it does not manage to obtain any market power.

Our analysis allows us to draw the following policy implications. First, it is not clear a priori how consumers are affected by changes in privacy rules. We show indeed that modifying these rules, and thereby affecting firms' ability to practice differential pricing, have an ambiguous impact on consumers. Take, for instance, the impact of less protective rules: on the one hand, some consumers will pay higher prices because firms can profile them more easily; but on the other hand, some consumers who used to be anonymous will now be profiled and among them, those with a low valuation will start purchasing and will thus enjoy a larger surplus; we even show the possibility that the positive impact on the latter consumers may be sufficiently important to outweigh the negative impact on the former ones, so that improved profiling ends up increasing consumer surplus on aggregate. The complex interplay between these countervailing forces may explain why the United States and the European Union have recently taken contrasting decisions regarding the protection of consumers privacy ${ }^{28}$

Although we suggest that the effects of strengthening or relaxing privacy protection are hard to ascertain, we are confident that all consumers should be better off if firms had the obligation to make their uniform prices public. With such observable uniform prices, firms would face an upper bound on the personalized prices they can charge to the consumers they profile, which is likely to drive prices down (possibly back to marginal costs).

Second, our results shed new light on the working of markets for data, which is also a heavily debated topic. Currently, the focus is essentially on the supply side of data, with concerns about potential barriers to entry stemming from the presence of economies of scale and/or of scope at the successive stages of the 'data value chain' (i.e., collection, storage, analysis, and usage) 29 Yet, conduct and strategies on the supply side of the markets for

\footnotetext{
${ }^{28}$ In the U.S., recent regulatory changes contribute to make customer data even more widely available to firms. In October 2016, the US Federal Communications Commission, then under Democratic majority, imposed a set of privacy rules on Internet service providers, requiring them to get opt-in consent from consumers before using, sharing, or selling their Web browsing data, app usage history and other private information. Yet, on April 3 2017, President Donald Trump signed a repeal of these rules, following actions taken by both houses of the US Congress (see, e.g., Brodkin (2017)). As for the E.U., it adopted in April 2016 the General Data Protection Regulation (GDPR), which is meant to strengthen and unify data protection for all individuals within the EU; this regulation will become directly applicable in all Member States from 25 May 2018.

${ }^{29}$ See, e.g., Rubinfeld and Gal (2017) and Duch-Brown et al. (2017).
} 
data cannot be properly analyzed without a good understanding of the demand side of these markets. In this respect, our contribution is to show that firms' willingness to pay for data (for price discrimination purposes) heavily depends on the profiling ability that this data confers and, more importantly, on what kind of customer analytics can be accessed by competitors. As a consequence, data owners/brokers have incentives to provide several firms with data as long as firms end up with different abilities to profile consumers; that is, some form of 'vertical data differentiation' arises. Regarding the regulation of markets for data, we note that exclusivity contracts offered by data brokers do not necessarily harm consumers. Indeed, in our model exclusivity leads to more - rather than less - competition.

In terms of empirical implications, we establish that when there is uncertainty regarding the nature of price competition, firms may randomize both their uniform and personalized prices. Then, there may be three simultaneous explanations as to why two consumers end up paying different prices: firms may charge them either different personalized prizes (price discrimination), or randomized uniform prices (price dispersion), or randomized personalized prices (price discrimination and dispersion) ${ }^{30}$ This provides a justification to the 'Amazon story'. In September 2000, a customer of Amazon.com accused Amazon of price discrimination (a.k.a. differential pricing): this customer realized that after having deleted the cookie that identified him as a regular Amazon customer, he was offered some DVD for a much lower price than the one he observed the first time he visited the web site. The company later apologized and explained that the price difference was not the result of differential pricing but of random price testing. ${ }^{31}$ Our results suggest that both Amazon and its customer may have been right. Meanwhile, a recent report by Bourreau et al. (2017) shows that although there is a lack of evidence of price discrimination, a large volatility of prices is observed. A related implication of our results is that the absence of direct observation of price discrimination should not be taken as proof that such a pricing tactic is not practiced, as it cannot be distinguished from price dispersion in some

\footnotetext{
${ }^{30}$ Our paper establishes thus a novel source of price dispersion. In the existing literature, price dispersion is attributed, for example, to search costs on the buyer's side (Varian (1980) and Burdett and Judd (1983), strategic advertising (Ireland (1993)), differences in the cost structure on the seller's side (Spulber (1995)), uncertainty about the number of active firms on the market (Janssen and Rasmusen (2002)), firms adding complexity to their price structures (Carlin (2009)), or uniform price constraints on the seller (Gautier and Wauthy (2010)). In most of these papers, price dispersion arises in equilibrium due to the uncertainty about the intensity of competition in the market. Our analysis, however, emphasizes that it is the uncertainty about the nature of competition that may generate market power and price dispersion of both uniform and personalized prices.

${ }^{31}$ See Ramasastry (2005).
} 
cases. In particular, when the nature of price competition is uncertain, it would be useful to account for all sources of price variations empirically, not just some of them.

\section{References}

Acquisti, A., C. Taylor, and L. Wagman (2016). The economics of privacy. Journal of Economic Literature 54(2), 442-92.

Belleflamme, P. and W. Vergote (2016). Monopoly price discrimination and privacy: The hidden cost of hiding. Economics Letters 149, 141-144.

BenMark, G., S. Klapdor, M. Kullmann, and R. Sundararajan (2017). How retailers can drive profitable growth through dynamic pricing. McKinsey \&6 Company (March). http://www.mckinsey.com/industries/retail/ourinsights/how-retailers-can-drive-profitable-growth-through-dynamicpricing.

Bourreau, M., A. de Streel, and I. Graef (2017). Big data and competition policy: Market power, personalised pricing and advertising. CERRE Final Report.

Braulin, F. C. and T. Valletti (2016). Selling customer information to competing firms. Economics Letters 149, 10-14.

Brodkin, J. (2017). President trump delivers final blow to web browsing privacy rules. Ars Technica (April 4). https://arstechnica.com/techpolicy/2017/04/trumps-signature-makes-it-official-isp-privacy-rules-are$\operatorname{dead} /$.

Burdett, K. and K. L. Judd (1983). Equilibrium price dispersion. Econometrica 51(4), 955-969.

Business Wire (2015). Ant financial unveils China's first creditscoring system using online data. Business Wire (January 27). http://www.businesswire.com/news/home/20150127006582/en/ Ant-Financial-Unveils-China\{2019\}s-Credit-Scoring-System-Online.

Calzolari, G. and A. Pavan (2006). On the optimality of privacy in sequential contracting. Journal of Economic theory 130(1), 168-204.

Carlin, B. I. (2009). Strategic price complexity in retail financial markets. Journal of financial Economics 91 (3), 278-287. 
Chen, Y. and G. Iyer (2002). Consumer addressability and customized pricing. Marketing Science 21(2), 197-208.

Choe, C., S. King, and N. Matsushima (2018). Pricing with cookies: Behavior-based price discrimination and spatial competition. Management Science 64(12), 5669-5687.

Corts, K. S. (1998). Third-degree price discrimination in oligopoly: all-out competition and strategic commitment. RAND Journal of Economics, 306-323.

Duch-Brown, N., B. Martens, and F. Mueller-Langer (2017). The economics of ownership, access and trade in digital data. JRC Digital Economy Working Paper 2017-01. https://ec.europa.eu/jrc/sites/jrcsh/files/jrc104756.pdf.

Encaoua, D. and A. Hollander (2007). First-degree discrimination by a duopoly: pricing and quality choice. The BE Journal of Theoretical Economics 7(1), Article 14.

European Insurance and Occupational Pensions Authority (2019). Big data analytics in motor and health insurance: A thematic review. https://eiopa.europa.eu/Publications/EIOPA_BigDataAnalytics_ ThematicReview_April2019.pdf.

Executive Office of the President of the United States (2015). Big data and differential pricing. Council of Economic Advisors, Washington, D.C. https://obamawhitehouse.archives.gov/sites/default/files/ whitehouse_files/docs/Big_Data_Report_Nonembargo_v2.pdf.

Feld, S., H. Frenzen, M. Krafft, K. Peters, and P. C. Verhoef (2013). The effects of mailing design characteristics on direct mail campaign performance. International Journal of Research in Marketing 30(2), 143-159.

Gautier, A. and X. Wauthy (2010). Price competition under universal service obligations. International Journal of Economic Theory 6(3), 311-326.

Ireland, N. J. (1993). The provision of information in a Bertrand oligopoly. Journal of Industrial Economics, 61-76.

Iyer, G., D. Soberman, and J. M. Villas-Boas (2005). The targeting of advertising. Marketing Science 24(3), 461-476.

Janssen, M. and E. Rasmusen (2002). Bertrand competition under uncertainty. Journal of Industrial Economics 50(1), 11-21. 
Jentzsch, N., G. Sapi, and I. Suleymanova (2013). Targeted pricing and customer data sharing among rivals. International Journal of Industrial Organization 31(2), 131-144.

Kehoe, P. J., B. J. Larsen, and E. Pastorino (2018). Dynamic competition in the era of big data. Technical report, Working paper, Stanford University and Federal Reserve Bank of Minneapolis.

Kim, J.-H., L. Wagman, and A. L. Wickelgren (2019). The impact of access to consumer data on the competitive effects of horizontal mergers and exclusive dealing. Journal of Economics \& Management Strategy 28(3), 373-391.

Liu, Q. and K. Serfes (2004). Quality of information and oligopolistic price discrimination. Journal of Economics \& Management Strategy 13(4), 671702.

McKinsey\&Company (2016). The age of analytics: Competing in a data-driven world. McKinsey Global Institute (December). https://www.mckinsey.com/business-functions/mckinsey-analytics/ourinsights/the-age-of-analytics-competing-in-a-data-driven-world.

Mikians, J., L. Gyarmati, V. Erramilli, and N. Laoutaris (2012). Detecting price and search discrimination on the internet. In Proceedings of the 11th ACM workshop on hot topics in networks, pp. 79-84.

Montes, R., W. Sand-Zantman, and T. Valletti (2019). The value of personal information in online markets with endogenous privacy. Management Science 65(3), 1342-1362.

Newman, N. (2013). The costs of lost privacy: Consumer harm and rising economic inequality in the age of Google. William Mitchell Law Review 40, 849.

Quito, A. and A. Shendruk (2019). US hospitals are now required by law to post prices online. Good luck finding them. Quartz (January 15). https://qz.com/1518545/price-lists-for-the-115-biggest-us-hospitalsnew-transparency-law/.

$\begin{gathered}\text { Ramasastry, A. (2005). Web sites change prices } \\ \text { based on customers' habits. }\end{gathered}$
http://edition.cnn.com/2005/LAW/06/24/ramasastry.website.prices/.


Rubinfeld, D. L. and M. S. Gal (2017). Access barriers to big data. Arizona Law Review 59, 339.

Shaffer, G. and Z. J. Zhang (2002). Competitive one-to-one promotions. Management Science 48(9), 1143-1160.

Shiller, B. (2014). First-degree price discrimination using big data. Working Paper 58, Brandeis University.

Spulber, D. F. (1995). Bertrand competition when rivals' costs are unknown. Journal of Industrial Economics, 1-11.

Taylor, C. and L. Wagman (2014). Consumer privacy in oligopolistic markets: Winners, losers, and welfare. International Journal of Industrial Organization 34, 80-84.

Thisse, J.-F. and X. Vives (1988). On the strategic choice of spatial price policy. American Economic Review, 122-137.

Valletti, T. and J. Wu (2016). 'Big Data' vs 'Small Data': Consumer profiling with data requirements. Technical report, Mimeo.

Varian, H. R. (1980). A model of sales. American Economic Review 70(4), 651-659.

Villas-Boas, J. M. (1999). Dynamic competition with customer recognition. Rand Journal of Economics, 604-631.

Wattal, S., R. Telang, T. Mukhopadhyay, and P. Boatwright (2012). What's in a "name"? Impact of use of customer information in e-mail advertisements. Information Systems Research 23(3), 679-697. 


\section{Appendix}

\subsection{Proof of Proposition 1}

The proof of this proposition is divided in 3 steps. In Step 1 we show under which conditions on the parameters there exists a pure strategy equilibrium and characterize that Nash equilibrium. In Step 2 we characterize the unique mixed strategy equilibrium when $(1-a) p_{A}^{x}<(1-b) p_{B}^{x}$ and determine the expected payoff for each firm. Finally, in step 3, we show that when $(1-a) p_{A}^{x}=(1-b) p_{B}^{x}$ two mixed strategy equilibria exist with an expected payoff for each firm equal to $(1-a) p_{A}^{x}=(1-b) p_{B}^{x}$. We therefore say that the mixed strategy equilibrium is essentially unique.

Remark: The reader will note that our proof of steps 1 and 2 is done for the case when $(1-a) p_{A}^{x}<(1-b) p_{B}^{x}$ and that the construction of the mixed strategy equilibrium does not depend on our assumption that $a>b$. In other words the equilibrium we constructed does not depend on the identity of the firm $A$ or $B$ : If $(1-a) p_{A}^{x}>(1-b) p_{B}^{x}$ we can just obtain an equivalent proposition by interchanging $p_{A}$ and $p_{B}$. We will make use of this in Step 3 to characterize the mixed strategy equilibria when $(1-a) p_{A}^{x}=(1-b) p_{B}^{x}$.

\section{Step I. Pure vs Mixed strategy equilibria}

To guide the reader we illustrate the ranges for which there exists a pure strategy or mixed strategy equilibrium in Figure 1.

Figure 1: Mixed vs Pure Strategy Nash Equilibrium.
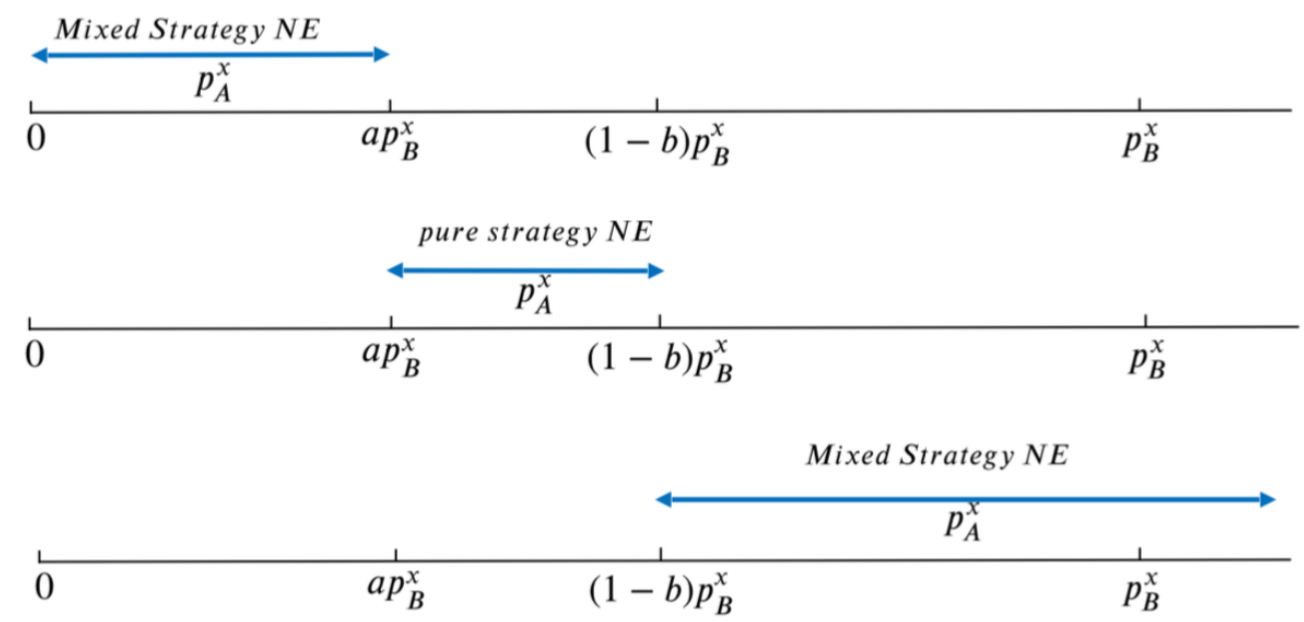

Lemma 3. Case I. When $p_{A}^{x}<(1-b) p_{B}^{x}$ we need to distinguish two cases:

1. No pure strategy Nash equilibrium exists when $p_{A}^{x}<a p_{B}^{x}$.

2. There is a pure strategy Nash equilibrium when $a p_{B}^{x} \leq p_{A}^{x} \leq(1-b) p_{B}^{x}$. In this Nash equilibrium the strategies are $\left(p_{A}(x), p_{B}(x)\right)=\left(p_{B}^{x}, p_{A}^{x}\right)$.

Case II. No pure strategy Nash equilibrium exists when $p_{A}^{x} \geq(1-b) p_{B}^{x}$. 
Proof. Proof of Lemma 3.

Case I. When $p_{A}^{x}<(1-b) p_{B}^{x}$.

Firm $A$ knows it can lure consumer $x$ into buying as long as $p_{A}(x) \leq p_{B}^{x}$. Firm $A$ will thus never charge a targeted price, $p_{A}(x)$, below $(1-b) p_{B}^{x}$ since this is the expected payoff from charging $p_{A}(x)=p_{B}^{x}$. Nor does it make sense to sell at a price above the consumer's willingness to pay: $p_{A}(x) \leq r(x)$. Now suppose that there exists a pure strategy equilibrium in which firm $A$ charges $p_{A}(x)$ such that $(1-b) p_{B}^{x} \leq p_{A}(x) \leq r(x)$. What is firm $B$ 's best response when she recognizes consumer $x$ ? Firm $B$ can either try to obtain the consumer $x$ with probability 1 by charging $p_{B}(x) \leq p_{A}^{x}$ or set at a higher price and sell to the consumer with probability $a$ by just undercutting $p_{A}(x)$. The first strategy yields profit $p_{A}^{x}$ while the second yields profit $a p_{A}(x)$. When $a p_{A}(x) \leq p_{A}^{x}$ firm $B$ 's best response is to play $p_{B}(x)=p_{A}^{x}$, when $a p_{A}(x)>p_{A}^{x}$ firm $B$ does not have a best response (wants to just undercut firm $A$, but because prices are chosen from a continuum, there is no best response). So in any pure strategy equilibrium it must be that $p_{B}(x)=p_{A}^{x}$, but then the best response of firm $A$ is to set $p_{A}(x)=p_{B}^{x}$. To sum up, for $\left(p_{A}(x), p_{B}(x)\right)=\left(p_{B}^{x}, p_{A}^{x}\right)$ to be a pure strategy equilibrium it must be that $a p_{B}^{x} \leq p_{A}^{x}$. We also have that $p_{A}^{x} \leq(1-b) p_{B}^{x}$, so a pure strategy Nash equilibrium can only occur when if $a \leq 1-b$. This is ruled out when $a+b>1$. In this pure strategy equilibrium firm $A$ 's expected payoff is $(1-b) p_{B}^{x}$ while firm $B$ 's expected payoff is $p_{A}^{x}$.

Case II. Mixed strategy Personalized pricing when $p_{A}^{x} \geq(1-b) p_{B}^{x}$.

Proof. We follow the same reasoning as Case 1. Again, firm $A$ will never set $p_{A}(x)<$ $(1-b) p_{B}^{x}$ or $p_{A}(x)>r(x)$. Suppose that there exists a pure strategy equilibrium in which firm $A$ charges $p_{A}(x)$ such that $r(x) \geq p_{A}(x) \geq(1-b) p_{B}^{x}$. What is firm $B$ 's best response when she recognizes consumer $x$ ?

First let $p_{A}(x) \geq p_{A}^{x}$. Then firm $B$ can either sell to consumer $x$ with probability 1 by charging $p_{B}(x) \leq p_{A}^{x}$ or set a higher price and sell to the consumer with probability $a$ by just undercutting $p_{A}(x)$. The first strategy yields profit $p_{A}^{x}$ while the second yields profit $a p_{A}(x)$. Hence when $a p_{A}(x) \leq p_{A}^{x}$ firm $B$ 's best response is to play $p_{B}(x)=p_{A}^{x}$, and when $a p_{A}(x)>p_{A}^{x}$ firm $B$ does not have a best response. So in any pure strategy equilibrium it must be that $p_{B}(x)=p_{A}^{x}$. Then the best response of firm $A$ would be to set $p_{A}(x)=p_{B}^{x}$ or to slightly undercut $p_{B}(x)=p_{A}^{x}$. Firm $A$ will choose $p_{A}(x)=p_{B}^{x}$ when $(1-b) p_{B}^{x}>p_{A}^{x}$. This is a contradiction since we assumed that $p_{A}^{x} \geq(1-b) p_{B}^{x}$.

Now let $p_{A}(x)<p_{A}^{x}$. Then firm $B$ can either try to sell to consumer $x$ with probability 1 by charging $p_{B}(x)<p_{A}(x)$ or set a higher price and sell to the consumer with probability $1-a$ by charging $p_{A}^{x}$. The first strategy yields a profit arbitrarily close to $p_{A}(x)$ while the second yields profit $(1-a) p_{A}^{x}$. Hence when $p_{A}(x) \leq(1-a) p_{A}^{x}$ firm $B$ 's best response is to play $p_{B}(x)=p_{A}^{x}$, and when $p_{A}(x) \geq(1-a) p_{A}^{x}$ firm $B$ does not have a best response. So in any pure strategy equilibrium it must be that $p_{B}(x)=p_{A}^{x}>p_{A}(x)$. But then firm $A$ has an incentive to increase the targeted price anywhere between $\left(p_{A}(x), p_{A}^{x}\right)$. As a consequence there cannot be any pure strategy equilibrium.

\section{Step II. Characterizing the mixed strategy Nash equilibria}

We now characterize the mixed strategy equilibrium and obtain expected payoffs for each firm in equilibrium. We first introduce a series of useful results that will help us construct the equilibrium strategies. We emphasize that none of the arguments we make 
to characterize the equilibrium strategies depends on our assumption that firm $A$ is better at recognizing consumers than firm $(a \geq b)$. We will focus on finding the mixed strategy equilibria when $(1-a) p_{A}^{x} \leq(1-b) p_{B}^{x}$, but by doing so we also characterize the mixed strategy Nash equilibria when $(1-a) p_{A}^{x} \geq(1-b) p_{B}^{x}$.

Lemma 4. Assume that $(1-a) p_{A}^{x}<(1-b) p_{B}^{x}$.

- Then Firm $A$ will never set a price below $(1-b) p_{B}^{x}$ in equilibrium and can always guarantee herself an expected payoff of $(1-b) p_{B}^{x}$.

- For firm $B$ we have:

- If $p_{A}^{x} \leq a(1-b) p_{B}^{x}$ firm $B$ will never set a price below $(1-b) p_{B}^{x}$ in equilibrium and can always guarantee herself an expected payoff of $a(1-b) p_{B}^{x}$.

- If $a(1-b) p_{B}^{x} \leq p_{A}^{x} \leq(1-b) p_{B}^{x}$ firm $B$ will never set a price below $p_{A}^{x}$ in equilibrium and can always guarantee herself an expected payoff of $p_{A}^{x}$.

- If $p_{A}^{x} \geq(1-b) p_{B}^{x}$ firm $B$ will never set a price below $(1-b) p_{B}^{x}$ in equilibrium and can always guarantee herself an expected payoff of $(1-b) p_{B}^{x}$.

Proof. Proof. The result for firm $A$ follows from the proof of lemma 3 above. The same logic yields the result for firm $B$.

Lemma 5. Let $p_{i}(x)$ be the infimum of all the equilibrium prices charged to consumer $x$ by firm $i=A, B$. If $\underline{p}_{A}(x)=\underline{p}_{B}(x)=\underline{p}(x)$ then no firm attaches positive probability to $\underline{p}(x)$.

Proof. Suppose firm $i$ attaches positive probability to $p(x)$. Now consider a targeted price $p_{j}(x)$ for firm $j$ arbitrarily close to $p(x)$ then firm $j$ can deviate (and increase her payoff) by slightly undercutting $\underline{p}(x)$, as she will add a strictly positive mass of sales (that she 'stole' from firm $i$ ).

We conclude from lemma 5 that if one firm plays the lowest price with positive probability then the other firm's lowest price must be strictly higher. We now determine the upper bound on prices in any equilibrium. This will allow us to pin down expected profits in any equilibrium.

Lemma 6. Let $\bar{p}_{i}(x)$ be the supremum of all the equilibrium prices charged to consumer $x$ by firm $i=A, B$. Let $\bar{p}(x)=\max \left\{\bar{p}_{A}(x), \bar{p}_{B}(x)\right\}$. When $(1-a) p_{A}^{x}<(1-b) p_{B}^{x}$, then $\bar{p}_{B}(x) \leq \bar{p}_{A}(x)=\bar{p}(x)=p_{B}^{x}$.

Proof. Proof. We first show that $\bar{p}(x) \leq p_{B}^{x}$. Suppose not, then either $\bar{p}_{A}(x)>p_{B}^{x}$ or $\bar{p}_{B}(x)>p_{B}^{x}$, or both.

Suppose first that $\bar{p}_{A}(x)>p_{B}^{x}$. Since $A$ will not sell to consumers it does not recognize it must be the case that firm $B$ sets prices equal to or higher than $\bar{p}_{A}(x)$ with positive probability: $\bar{p}_{B}(x) \geq \bar{p}_{A}(x)$. Firm $B$ will only be willing to do so if $\bar{p}_{B}(x) \leq p_{A}^{x}$. By lemma 4 , firm $B$ can guarantee a payoff of $(1-b) p_{B}^{x}$. By playing $\bar{p}_{B}(x)$ firm $B$ obtains strictly less, a contradiction:

$$
(1-a) \bar{p}_{B}(x) \leq(1-a) p_{A}^{x}<(1-b) p_{B}^{x} .
$$


Now suppose that $\bar{p}_{B}(x)>\bar{p}(x) \geq \bar{p}_{A}(x)$. Again it must be that $\bar{p}_{B}(x) \leq p_{A}^{x}$, otherwise firm $B$ would obtain zero expected profit from setting its price equal (or close) to its supremum $\bar{p}_{B}(x)$. We obtain the same contradiction:

$$
(1-a) \bar{p}_{B}(x) \leq(1-a) p_{A}^{x}<(1-b) p_{B}^{x} .
$$

We now show that $\bar{p}(x) \geq p_{B}^{x}$. First note that one cannot have that $\bar{p}_{B}(x)<\bar{p}_{A}(x)<$ $p_{B}^{x}$ since otherwise firm $A$ can increase profits by setting $\bar{p}_{A}(x)=p_{B}^{x}$. Then suppose that $\bar{p}_{A}(x) \leq \bar{p}_{B}(x)<p_{B}^{x}$. In order for firm $A$ to obtain profits equal to $(1-b) p_{B}^{x}$, firm $B$ must assign positive mass to prices from the set $\left[\bar{p}_{A}(x), \bar{p}_{B}^{x}\right]$ and $\bar{p}_{B}(x) \leq p_{A}^{x}$. This would imply that if $\bar{p}_{A}(x)<\bar{p}_{B}^{x}$ firm $B$ will only play $\bar{p}_{B}(x)$ from this interval. But then firm $A$ can just increase her price slightly above $\bar{p}_{A}(x)$, not lose any customers and increase her expected profit. Hence the only possibility left is $\bar{p}_{B}(x)=\bar{p}_{A}(x)<p_{B}^{x}$. Then $B$ must play $\bar{p}_{B}(x)$ with positive probability so that firm $A$ can guarantee herself a payoff of $(1-b) p_{B}^{x}$ while firm $A$ attaches zero probability to it. But then firm $B$ will only be willing to do so if $\bar{p}_{B}(x)=\bar{p}_{A}(x) \leq p_{A}^{x}$. By lemma 4 , firm $B$ can guarantee a payoff of $(1-b) p_{B}^{x}$. By playing $\bar{p}_{B}(x)$ firm $B$ obtains strictly less, a contradiction.

Lemma 7. Let $\bar{p}(x)$ be the supremum of all the equilibrium prices charged to consumer $x$ by either firm. It cannot be that both firms play this price with positive probability.

Proof. This follows from the fact that both firms would have an incentive to slightly undercut the other.

Lemma 8. When $(1-a) p_{A}^{x}<(1-b) p_{B}^{x}$, the expected payoff for firm $A$ in any equilibrium is $(1-b) p_{B}^{x}$.

Proof. This follows immediately from the fact that equilibrium prices cannot be above $p_{B}^{x}$ and that firm $A$ is guaranteed a payoff of $(1-b) p_{B}^{x}$. To have a higher expected payoff, firm $B$ should play $p_{B}^{x}$ with positive probability. The payoff of firm $B$ is then $(1-a) p_{B}^{x}<(1-b) p_{B}^{x}$ if $p_{B}^{x} \leq p_{A}^{x}$. But in this case firm $B$ can guarantee herself an expected profit of $(1-b) p_{B}^{x}$, a contradiction. If on the other hand $p_{B}^{x}>p_{A}^{x}$, firm $B$ makes zero profits from playing $p_{B}^{x}$.

We are now ready to fully characterize the mixed strategy equilibrium. We need to consider several different cases.

Step II Case 1. $p_{A}^{x}<a(1-b) p_{B}^{x}$

Figure 2: Step II case 1

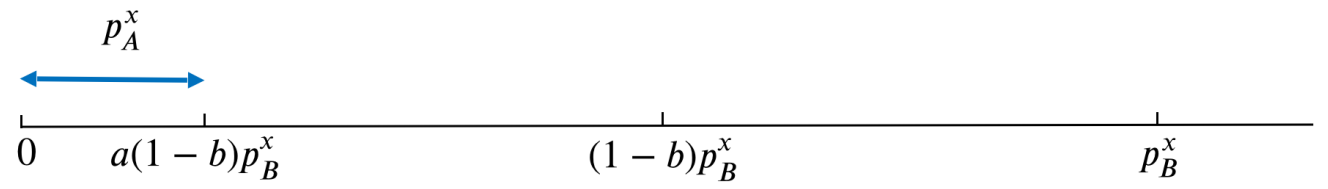


This case is illustrated in Figure 2. In this case firm $B$, like firm $A$, will not set a price below $(1-b) p_{B}^{x}$. They must set the same lowest price and this price cannot be a mass point of the price distribution of any firm (see Lemma 5). If one firm were to have a lowest price which is higher than the one of the other firm, then the latter has an incentive to deviate and increase her lowest price since it will not change her probability of selling to the consumer while at the same time it increases her sales price.

Let $F_{B}^{1}(p)$ be the probability distribution of prices of firm $B$ in equilibrium in case 1 . For firm $A$ the following must hold:

1. For any price $p_{A}(x)$ firm $A$ must get an expected payoff of $(1-b) p_{B}^{x}$. Then firm $A$ must have a probability of selling equal to one when charging $p_{A}(x)=(1-b) p_{B}^{x}$.

2. For any other price $p$ : $(1-b) p+b\left(1-F_{B}^{1}(p) p=(1-b) p_{B}^{x}\right.$.

3. It follows that

$$
F_{B}^{1}(p)=1-\frac{(1-b)\left(p_{B}^{x}-p\right)}{b p}
$$

Let $F_{A}^{1}(p)$ be the probability distribution of prices of firm $A$ in equilibrium in case 1 . For firm $B$ the following must hold:

1. For any price $p_{B}(x)$ charged firm $B$ must get an expected payoff of $a(1-b) p_{B}^{x}$. Thus firm $B$ must have a probability of selling equal to $a$ when charging $p_{B}(x)=(1-b) p_{B}^{x}$.

2. For any other price $p$ : $a\left(1-F_{A}(p)\right) p=a(1-b) p_{B}^{x}$,

$$
F_{A}^{1}(p)=1-\frac{(1-b) p_{B}^{x}}{p}
$$

3. Note that

$$
F_{A}^{1}\left(p_{B}^{x}\right)=b
$$

We conclude that firm $A$ must play $p_{A}(x)=p_{B}^{x}$ with probability $1-b$.

In summary, we obtain:

- Firm $A$ mixes over $\left((1-b) p_{B}^{x}, p_{B}^{x}\right]$ according to distribution $F_{A}^{1}(p)=1-\frac{(1-b) p_{B}^{x}}{p}$ where $F_{A}^{1}\left(p_{B}^{x}\right)=b$.

- Firm $B$ mixes over $\left((1-b) p_{B}^{x}, p_{B}^{x}\right)$ according to distribution $F_{B}^{1}(p)=1-\frac{(1-b)\left(p_{B}^{x}-p\right)}{b p}$.

Step II Case 2. $a(1-b) p_{B}^{x}<p_{A}^{x}<(1-b) p_{B}^{x}$

This case is illustrated in Figure 3. If $p_{A}^{x} \geq a p_{B}^{x}$ then there exists a pure strategy equilibrium, as argued above. We characterize the mixed strategy equilibrium in the event that $p_{A}^{x}<a p_{B}^{x}$. Again we will use the fact that firm $A$ must have an expected payoff in any equilibrium equal to $(1-b) p_{B}^{x}$ in order to pin down the (unique) equilibrium strategies. Let $F_{i}^{2}(p)$ be the probability distribution of prices of firm $i=A, B$ in equilibrium in case 2. We first state the equilibrium strategies after which we develop the proof:

- Firm $A$ mixes over $\left(\frac{p_{A}^{x}}{a}, p_{B}^{x}\right]$ 
Figure 3: Step II case 2

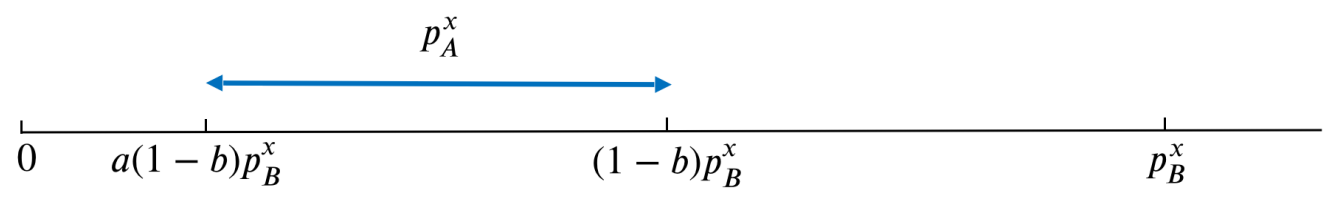

- She plays $p_{B}^{x}$ with probability $\Psi_{A}=\frac{p_{A}^{x}}{a p_{B}^{x}} \leq 1$ and plays any price on $\left(\frac{p_{A}^{x}}{a p_{B}^{x}}, p_{B}^{x}\right)$ according to distribution function $F_{A}^{2}(p)=1-\frac{p_{A}^{x}}{a p}$.

- Firm $B$ mixes over $p_{A}^{x}$ and $\left(\frac{p_{A}^{x}}{a}, p_{B}^{x}\right)$

- She plays $p_{A}^{x}$ with probability $\Psi_{B}=\frac{1}{b}-\frac{a(1-b) p_{B}^{x}}{b p_{A}^{x}}$ and plays any price on $\left(\frac{p_{A}^{x}}{a p_{B}^{x}}, p_{B}^{x}\right)$ according to $F_{B}^{2}(p)=F_{B}^{2}(p)=\frac{1}{b}-\frac{(1-b) p_{B}^{x}}{b p}$.

Since firm $A$ obtains expected profit equal to $(1-b) p_{B}^{x}$ in any equilibrium, firm $A$ will not set a price below $(1-b) p_{B}^{x}$ while the supremum of its equilibrium prices is $p_{B}^{x}$ in any mixed strategy equilibrium. Now imagine that firm $A$ and firm $B$ would charge prices equal to or slightly above $(1-b) p_{B}^{x}$ in equilibrium. For firm $B$ this would mean that she would obtain an expected payoff of approximately $a(1-b) p_{B}^{x}$. Then firm $B$ would have a profitable deviation by lowering her price to $p_{A}^{x}$ and obtain $p_{A}^{x}>a(1-b) p_{B}^{x}$. Indeed, she is better off selling to customer with probability 1 by charging $p_{A}^{x}$ than selling to only those consumers that firm $A$ also recognizes (with probability $a$ ) as this yields $a(1-b) p_{B}^{x} \leq p_{A}^{x}$. But if firm $B$ will not charge prices in neighborhood of $(1-b) p_{B}^{x}$, firm $A$ will not play these prices as she can simply increase her price, not lose any consumers and increase her expected payoff. In other words, $(1-b) p_{B}^{x}$ cannot be the lowest price that firm $A$ charges in equilbrium. What then will this lowest price, $\underline{p}_{A}(x)$, be? Following the above logic, the lowest price firm $A$ charges in equilibrium, $p_{A}(x)$, must make firm $B$ indifferent between obtaining the consumer for sure and charging $\underline{p}_{A}(x)$, obtaining payoff $a \underline{p}_{A}(x)$. We now detail this intuition.

Since firm $A$ must be playing a mixed strategy it must be indifferent between the (infimum of the) lowest price it charges, $\underline{p}_{A}(x)$, and the (supremum of the) highest price it charges, $\bar{p}_{A}(x)=p_{B}^{x}$. On the other hand firm $B$ is also playing a mixed strategy and should be indifferent between playing $p_{A}^{x}$ and any other equilibrium price $p_{B}(x)$ price. But then the lowest price firm $B$ is willing to charge above $p_{A}^{x}$, call it $\underline{p}_{B}(x)$, must guarantee her a payoff of $p_{A}^{x}$. This is the case when $a \underline{p}_{B}(x)=p_{A}^{x}$ for some $\underline{p}_{B}(x) \leq p_{B}^{x}$. Hence $\underline{p}_{B}(x)=\frac{p_{A}^{x}}{a}$. It then follows that $\underline{p}_{B}(x)=\underline{p}_{A}(x)=\frac{p_{A}^{x}}{a}$.

For firm $A$ to be indifferent between $\underline{p}_{A}(x)=\frac{p_{A}^{x}}{a}$ and $p_{B}^{x}$ it must be that firm $B$ charges $p_{A}^{x}$ with probability $\Psi_{B}$ where

$$
(1-b) \frac{p_{A}^{x}}{a}+b\left(1-\Psi_{B}\right) \frac{p_{A}^{x}}{a}=(1-b) p_{B}^{x}
$$


Hence $\Psi_{B}=\frac{1}{b}-\frac{a(1-b) p_{B}^{x}}{b p_{A}^{x}}$. Let $F_{B}^{2}(p)$ be the probability distribution of prices of firm $B$ drawn from $\left(\frac{p_{A}^{x}}{a}, p_{B}^{x}\right]$. For firm $A$ to be indifferent between any price $p \in\left(\frac{p_{A}^{x}}{a}, p_{B}^{x}\right]$ it must be that

$$
\begin{gathered}
(1-b) p+b\left(1-F_{B}^{2}(p)\right) p=(1-b) p_{B}^{x}, \\
F_{B}^{2}(p)=\frac{1}{b}-\frac{(1-b) p_{B}^{x}}{b p} .
\end{gathered}
$$

Note that $F_{B}^{2}\left(\frac{p_{A}^{x}}{a}\right)=\frac{1}{b}-\frac{(1-b) p_{B}^{x}}{b \frac{p_{A}^{x}}{a}}=\Psi_{B}$ and $F_{B}^{2}\left(p_{B}^{x}\right)=\frac{1}{b}-\frac{(1-b) p_{B}^{x}}{b p_{B}^{x}}=\frac{1}{b}-\frac{(1-b)}{b}=1$. Firm $B$ must also be indifferent between any price it charges given the mixed strategy chosen by firm $A$. Let $F_{A}^{2}(p)$ be the probability distribution of prices of firm $A$ drawn from $\left(\frac{p_{A}^{x}}{a}, p_{B}^{x}\right]$. For firm $B$ to be indifferent between any price $p \in\left(\frac{p_{A}^{x}}{a}, p_{B}^{x}\right)$ it must be that

$$
\begin{gathered}
a\left(1-F_{A}^{2}(p)\right) p=p_{A}^{x}, \\
F_{A}^{2}(p)=1-\frac{p_{A}^{x}}{a p} .
\end{gathered}
$$

This must imply that firm $A$ plays $p_{A}(x)=p_{B}^{x}$ with positive probability $\Psi_{A}$ equal to

$$
\Psi_{A}=1-\left(1-\frac{p_{A}^{x}}{a p_{B}^{x}}\right)=\frac{p_{A}^{x}}{a p_{B}^{x}}<1 .
$$

Step II Case 3. $p_{A}^{x} \geq(1-b) p_{B}^{x}$

This last case is illustrated in Figure 4:

Figure 4: Step II case 3

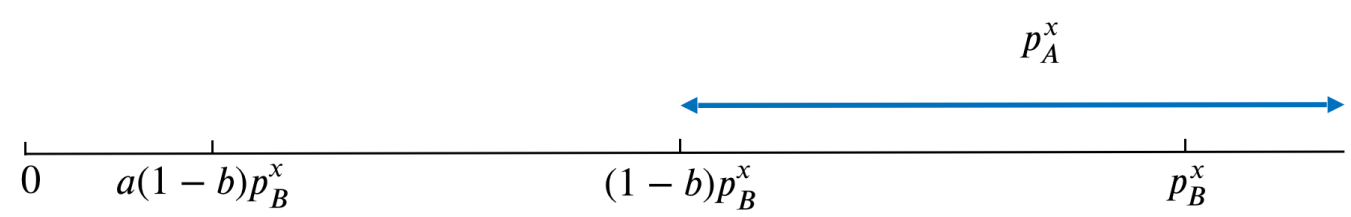

We need to consider two subcases:

- Step II Case $3 \mathbf{i}(1-b) p_{B}^{x} \leq p_{A}^{x}<\frac{(1-b) p_{B}^{x}}{2-a-b}$.

- Step II Case 3ii $\frac{(1-b) p_{B}^{x}}{2-a-b} \leq p_{A}^{x}<\frac{(1-b) p_{B}^{x}}{1-a}$.

Step II Case 3i Let $F_{j}^{3 i}(p)$ be the probability distribution of prices of firm $j=A, B$ in case $3 i$ in equilibrium in case $3 i$. When $(1-b) p_{B}^{x} \leq p_{A}^{x}<\frac{(1-b) p_{B}^{x}}{2-a-b}$ we will prove the following:

- Firm $B$ mixes its price $p_{B}(x)$ according to the distribution function $F_{B}^{3 i}(p)$ over the support:

$$
\left[(1-b) p_{B}^{x}, p_{A}^{x}\right] \cup\left[\tilde{p}(x), p_{B}^{x}\right)
$$

and plays price $p=p_{A}^{x}$ with probability $\Phi_{B}=\frac{1-b}{a}$. 
- Firm $A$ mixes its price $p_{A}(x)$ according to the distribution function $F_{A}^{3 i}(p)$ over the support:

$$
\left[(1-b) p_{B}^{x}, p_{A}^{x}\right) \cup\left[\tilde{p}(x), p_{B}^{x}\right]
$$

and plays price $p=p_{B}^{x}$ with probability $\Phi_{A}=\frac{1-a}{b}$.

- For $p \in\left((1-b) p_{B}^{x}, p_{A}^{x}\right)$ we have that $F_{A}^{3 i}(p)=1-\frac{(1-b) p_{B}^{x}-(1-a) p}{a p}$ and $F_{B}^{3 i}(p)=$ $1-\frac{(1-b)\left(p_{B}^{x}-p\right)}{b p}$.

- For $p \in\left[\tilde{p}(x), p_{B}^{x}\right)$ we have that $F_{A}^{3 i}(p)=1-\frac{(1-b) p_{B}^{x}}{a p}$ and $F_{B}^{3 i}(p)=1-\frac{(1-b)\left(p_{B}^{x}-p\right)}{b p}$.

The reasoning behind this case is similar to case 2. But now, if the infimum of the support of prices charged by firm $A$ is $(1-b) p_{B}^{x}$ then firm $B$ no longer wants to set a price below $(1-b) p_{B}^{x}$ in order to sell to consumer $x$ with probability 1 . However, when $p_{A}^{x} \geq(1-b) p_{B}^{x}$ another discontinuity of firm $B$ 's expected profit with respect to its targeted price potentially occurs at $p_{B}(x)=p_{A}^{x}$. In particular, whenever firm $B$ charges higher prices in equilibrium the support of price distributions for both firms cannot be connected. This happens when $(1-b) p_{B}^{x} \leq p_{A}^{x}<\frac{(1-b) p_{B}^{x}}{2-a-b}$. But then we ask: which price above $p_{A}^{x}$ and below $p_{B}^{x}$, call it $\tilde{p}(x)$, makes firm $B$ indifferent between charging this price, charging $p_{A}^{x}$ or charging $(1-b) p_{B}^{x}$ ? Then $\tilde{p}(x)$ must be such that

- Charging $p_{B}(x)=p_{A}^{x}$ must yield $(1-b) p_{B}^{x}:(1-a) p_{A}^{x}+a\left(1-F_{A}^{3 i}\left(p_{A}^{x}\right)\right) p_{A}^{x}=(1-b) p_{B}^{x}$,

- Charging $p_{B}(x)=\tilde{p}(x)$ must yield $(1-b) p_{B}^{x}: a\left(1-F_{A}^{3 i}(\tilde{p}(x)) \tilde{p}(x)=(1-b) p_{B}^{x}\right.$,

and imposing that $F_{A}^{3 i}\left(p_{A}^{x}\right)=F_{A}^{3 i}(\tilde{p}(x))$ we obtain that:

$$
\begin{gathered}
\left(1-a F_{A}^{3 i}\left(p_{A}^{x}\right)\right) p_{A}^{x}=(1-b) p_{B}^{x}, \\
F_{A}^{3 i}\left(p_{A}^{x}\right)=\frac{1}{a}-\frac{(1-b) p_{B}^{x}}{a p_{A}^{x}}=\frac{p_{A}^{x}-(1-b) p_{B}^{x}}{a p_{A}^{x}}=F_{A}^{3 i}(\tilde{p}(x)),
\end{gathered}
$$

we then have that

$$
1-F_{A}^{3 i}\left(p_{A}^{x}\right)=1-\frac{p_{A}^{x}-(1-b) p_{B}^{x}}{a p_{A}^{x}}=\frac{(1-b) p_{B}^{x}-(1-a) p_{A}^{x}}{a p_{A}^{x}} .
$$

We find $\tilde{p}(x)$ in the following way:

$$
\begin{gathered}
a\left(\frac{(1-b) p_{B}^{x}-(1-a) p_{A}^{x}}{a p_{A}^{x}}\right) \tilde{p}(x)=(1-b) p_{B}^{x} ; \\
\tilde{p}(x)=\frac{p_{A}^{x}(1-b) p_{B}^{x}}{(1-b) p_{B}^{x}-(1-a) p_{A}^{x}} .
\end{gathered}
$$

It must also be the case that firm $B$ obtains a payoff equal to $(1-b) p_{B}^{x}$ for any price it charges arbitrarily close to but below $p_{B}^{x}$. But then firm $A$ must play $p_{B}^{x}$ with positive probability, call it $\Phi_{A}$. We find that:

- Charging $p_{B}(x) \sim p_{B}^{x}$ must yield $(1-b) p_{B}^{x}: a \Phi_{A} p_{B}^{x}=(1-b) p_{B}^{x}$. This happens when $\Phi_{A}=\frac{1-b}{a}$.

Let us now switch to the equilibrium price conditions for firm $A$. 
- Charging $p_{A}(x) \sim p_{A}^{x}$ must yield $(1-b) p_{B}^{x}$ :

$$
\lim _{p_{A}(x) \rightarrow p_{A}^{x}}(1-b) p_{A}(x)+b\left(1-F_{B}^{3 i}\left(p_{A}(x)\right)\right) p_{A}(x)=(1-b) p_{B}^{x}
$$

or

$$
(1-b) p_{A}^{x}+b\left(1-\lim _{p_{A}(x) \rightarrow p_{A}^{x}} F_{B}^{3 i}\left(p_{A}(x)\right)\right) p_{A}(x)=(1-b) p_{B}^{x} .
$$

- Charging $p_{A}(x)=\tilde{p}(x)$ must yield $(1-b) p_{B}^{x}:(1-b) \tilde{p}(x)+b\left(1-F_{A}^{3 i}(\tilde{p}(x)) \tilde{p}(x)=\right.$ $(1-b) p_{B}^{x}$.

These conditions imply that firm $B$ must attach a positive probability to playing $p_{B}(x)=$ $p_{A}^{x}$ and firm $A$ will charge all prices close to and strictly below $p_{A}^{x}$ but price equal to $p_{A}^{x}$ (this interval of prices for $A$ is open at $p_{A}^{x}$ ) since there is a positive probability it will have to 'share' the consumer since firm $B$ plays this price with positive mass. Call this probability $\Phi_{B}$. We find that $\Phi_{A}=\frac{1-a}{b}$. What is left to determine are the probability distributions $F_{A}^{3 i}(p)$ and $F_{B}^{3}(p)$.

For $p \in\left((1-b) p_{B}^{x}, p_{A}^{x}\right)$ we have that:

$$
(1-a) p+a\left(1-F_{A}^{3 i}(p)\right) p=(1-b) p_{B}^{x} \text { and }(1-b) p+b\left(1-F_{B}^{3 i}(p)\right) p=(1-b) p_{B}^{x},
$$

hence

$$
F_{A}^{3 i}(p)=1-\frac{(1-b) p_{B}^{x}-(1-a) p}{a p} \text { and } F_{B}^{3 i}(p)=1-\frac{(1-b)\left(p_{B}^{x}-p\right)}{b p} .
$$

For $p \in\left[\tilde{p}(x), p_{B}^{x}\right)$ we have that:

$$
\left(1-F_{A}^{3 i}(p)\right) p=(1-b) p_{B}^{x} \text { and }(1-b) p+b\left(1-F_{B}^{3 i}(p)\right) p=(1-b) p_{B}^{x},
$$

hence

$$
F_{A}^{3 i}(p)=1-\frac{(1-b) p_{B}^{x}}{a p} \text { and } F_{B}^{3 i}(p)=1-\frac{(1-b)\left(p_{B}^{x}-p\right)}{b p} .
$$

In addition we have that

$$
\Phi_{A}=\frac{1-a}{b} \text { and } \Phi_{B}=\frac{1-b}{a} .
$$

Step II Case 3ii To finish the proof we still need to tackle the case when $\frac{(1-b) p_{B}^{x}}{2-a-b} \leq$ $p_{A}^{x} \leq \frac{(1-b) p_{B}^{x}}{1-a}$.

Let $F_{j}^{3 i i}(p)$ be the probability distribution of prices of firm $j=A, B$ in case $3 i i$ in equilibrium in case $3 i i$. This case is very similar to the previous one (when $(1-b) p_{B}^{x} \leq$ $\left.p_{A}^{x}<\frac{(1-b) p_{B}^{x}}{2-a-b}\right)$, but slightly easier. There are two sub-cases to consider: $p_{A}^{x}<p_{B}^{x}$ and $p_{A}^{x} \geq p_{B}^{x}$.

Note that when $p_{A}^{x}<p_{B}^{x}$ then there is no price $\tilde{p}(x)<p_{B}^{x}$ as defined above that would make firm $B$ indifferent between selling to the consumer only when firm $A$ also recognizes the consumer and selling at price $p_{A}^{x}$ guaranteeing to sell to the consumer in the event that firm $A$ does not recognize the consumer. We then have for $p \in\left((1-b) p_{B}^{x}, p_{A}^{x}\right)$ that:

$$
(1-a) p+a\left(1-F_{A}^{3 i i}(p)\right) p=(1-b) p_{B}^{x} \text { and }(1-b) p+b\left(1-F_{B}^{3 i i}(p)\right) p=(1-b) p_{B}^{x},
$$

hence

$$
F_{A}^{3 i i}(p)=1-\frac{(1-b) p_{B}^{x}-(1-a) p}{a p} \text { and } F_{B}^{3 i i}(p)=1-\frac{(1-b)\left(p_{B}^{x}-p\right)}{b p}
$$


and we equally find that

$$
\Phi_{A}^{3 i i}=\frac{(1-b) p_{B}^{x}-(1-a) p}{a p_{A}^{x}} \text { and } \Phi_{B}^{3 i i}=\frac{(1-b)\left(p_{B}^{x}-p_{A}^{x}\right)}{b p_{A}^{x}},
$$

where $\Phi_{A}^{3 i i}$ is the probability mass firm $A$ assigns to price $p_{B}^{x}$ and $\Phi_{B}^{3 i i}$ is the probability mass firm $B$ assigns to price $p_{A}^{x}$.

Finally in the case that $p_{A}^{x} \geq p_{B}^{x}$, the easiest case, the firms will randomize their prices over the interval $\left[(1-b) p_{B}^{x}, p_{B}^{x}\right)$ and in order for firm $B$ to obtain positive profits (equal to $\left.(1-b) p_{B}^{x}\right)$, firm $A$ will assign probability mass $\frac{a-b}{b}$ to price $p_{B}^{x}$ which is the supremum of the support of prices for both firms. For $p \in\left[(1-b) p_{B}^{x}, p_{B}^{x}\right)$ we have that:

$$
(1-a) p+a\left(1-F_{A}^{3 i i}(p)\right) p=(1-b) p_{B}^{x} \text { and }(1-b) p+b\left(1-F_{B}^{3 i i}(p)\right) p=(1-b) p_{B}^{x},
$$

hence

$$
F_{A}^{3 i i}(p)=1-\frac{(1-b) p_{B}^{x}-(1-a) p}{a p} \text { and } F_{B}^{3 i i}(p)=1-\frac{(1-b)\left(p_{B}^{x}-p\right)}{b p}
$$

\section{Step III. Mixed strategy Nash equilibria when $(1-a) p_{A}^{x}=(1-b) p_{B}^{x}$}

We now construct the equilibrium strategies of two mixed strategy equilibria when $(1-a) p_{A}^{x}=(1-b) p_{B}^{x}$. The two equilibria will be the ones obtained in the limit when $(1-a) p_{A}^{x}$ approaches $(1-b) p_{B}^{x}$ from below and from above. The expected payoff for both firms is equivalent in both equilibria and equal to $(1-a) p_{A}^{x}=(1-b) p_{B}^{x}$.

Equilibrium 1: When $(1-a) p_{A}^{x}$ approaches $(1-b) p_{B}^{x}$ from below the highest price played in equilibrium is anchored at $p_{B}^{x}$, a property which is preserved in the limit. Note that since $a \geq b$ we have that $p_{A}^{x} \geq p_{B}^{x}$. Hence the Nash equilibrium is the one that we have just described in the previous paragraph. the firms will randomize their prices over the interval $\left[(1-b) p_{B}^{x}, p_{B}^{x}\right)$ and in order for firm $B$ to obtain positive profits (equal to $\left.(1-b) p_{B}^{x}\right)$, firm $A$ will assign probability mass $\frac{a-b}{b}$ to price $p_{B}^{x}$ which is the supremum of the support of prices for both firms. For $p \in\left[(1-b) p_{B}^{x}, p_{B}^{x}\right)$ we have that:

$$
(1-a) p+a\left(1-F_{A}^{3 i i}(p)\right) p=(1-b) p_{B}^{x} \text { and }(1-b) p+b\left(1-F_{B}^{3 i i}(p)\right) p=(1-b) p_{B}^{x}
$$

hence

$$
F_{A}^{3 i i}(p)=1-\frac{(1-b) p_{B}^{x}-(1-a) p}{a p} \text { and } F_{B}^{3 i i}(p)=1-\frac{(1-b)\left(p_{B}^{x}-p\right)}{b p} .
$$

It is immediately checked that any individual deviation to any other price cannot strictly increase expected profit. Firm $B$ will not charge any price below $(1-b) p_{B}^{x}$ as this would yield lower expected utility. The most she can hope for from charging a higher price is $(1-a) p_{A}^{x}$, but this is equal to $(1-b) p_{B}^{x}$. If firm $A$ deviates to any other prices than prices in the interval $\left[(1-b) p_{B}^{x}, p_{B}^{x}\right]$ her expected profits will be lower than $(1-b) p_{B}^{x}$.

Equilibrium 2: When $(1-a) p_{A}^{x}$ approaches $(1-b) p_{B}^{x}$ from above the highest price played in equilibrium is anchored at $p_{A}^{x}$, a property which is again preserved in the limit.

To characterize this equilibrium we just need to switch the firms' labels in the above proof. It is the case that $p_{B}^{x} \geq(1-a) p_{A}^{x}$ and hence we are in case 3 of the above proof. In particular since

$$
\frac{(1-a) p_{A}^{x}}{2-a-b}=\frac{(1-b) p_{B}^{x}}{2-a-b} \leq p_{B}^{x} .
$$


We are in the second sub-case of case 3 while $p_{B}^{x} \leq p_{A}^{x}$, which is the yields the equilibrium of the first sub-case. We then have for $p \in\left((1-a) p_{A}^{x}, p_{B}^{x}\right)$ that:

$$
(1-b) p+a\left(1-F_{B}^{3 i i}(p)\right) p=(1-a) p_{A}^{x} \text { and }(1-a) p+a\left(1-F_{A}^{3 i i}(p)\right) p=(1-a) p_{A}^{x},
$$

hence

$$
F_{B}^{3 i i}(p)=1-\frac{(1-a) p_{A}^{x}-(1-b) p}{b p} \text { and } F_{A}^{3 i i}(p)=1-\frac{(1-a)\left(p_{A}^{x}-p\right)}{a p}
$$

and we equally find that

$$
\Phi_{B}^{3 i i}=\frac{(1-a) p_{A}^{x}-(1-b) p}{b p_{B}^{x}} \text { and } \Phi_{A}^{3 i i}=\frac{(1-a)\left(p_{A}^{x}-p_{B}^{x}\right)}{a p_{B}^{x}},
$$

where $\Phi_{B}^{3 i i}$ is the probability mass firm $B$ assigns to price $p_{A}^{x}$ and $\Phi_{A}^{3 i i}$ is the probability mass firm $A$ assigns to price $p_{B}^{x}$.

For the same reasons as equilibrium 1, it is immediately checked that any individual deviation to any other price cannot strictly increase expected profit.

Expected payoff. It follows immediately from the equilibrium strategies that the expected payoff for both firms is equal to

$$
(1-a) p_{A}^{x}=(1-b) p_{B}^{x}
$$

\subsection{Perfectly correlated profiling technologies}

\subsubsection{Proof of Proposition 3}

Stage 2. Denote by $\tilde{\pi}_{i}(p)$ firm $i$ 's expected profit obtained from consumer $x$ when pricing $p_{i}(x)=p$ at stage 2 . These profits are conditional on being on segments where personalized prices are relevant (i.e., the $A$-translucent and transparent segments for firm $A$, with probability $\lambda$, and the transparent segment for firm $B$, with probability $\beta \lambda$ ). Supposing that firm $B$ draws its price $p_{B}(x)$ from some distribution $G_{B}^{x}(p)$ that is defined on the interval $\left[(1-\beta) p_{B}^{x}, p_{B}^{x}\right]$, let us show that firm $A$ achieves the same expected profit when playing $p_{A}(x)=(1-\beta) p_{B}^{x}$ or $p_{A}(x)=p_{B}^{x}$. As $G_{B}^{x}\left((1-\beta) p_{B}^{x}\right)=0$ and $G_{B}^{x}\left(p_{B}^{x}\right)=1$, we have indeed that

$$
\begin{aligned}
\tilde{\pi}_{A}\left((1-\beta) p_{B}^{x}\right) & =(1-\beta)^{2} p_{B}^{x}+\beta\left(1-G_{B}^{x}\left((1-\beta) p_{B}^{x}\right)\right)(1-\beta) p_{B}^{x}=(1-\beta) p_{B}^{x}, \\
\tilde{\pi}_{A}\left(p_{B}^{x}\right) & =(1-\beta) p_{B}^{x}+\beta\left(1-G_{B}^{x}\left(p_{B}^{x}\right)\right) p_{B}^{x}=(1-\beta) p_{B}^{x} .
\end{aligned}
$$

For firm $A$ to be indifferent among any price $p \in\left[(1-\beta) p_{B}^{x}, p_{B}^{x}\right]$, its expected profit must always be equal to $(1-\beta) p_{B}^{x}$; that is $\tilde{\pi}_{A}(p)=(1-\beta) p+\beta\left(1-G_{B}^{x}(p)\right) p=(1-\beta) p_{B}^{x}$, from which we obtain

$$
G_{B}^{x}(p)=\frac{p-(1-\beta) p_{B}^{x}}{\beta p}=G^{x}(p) .
$$

Suppose now that firm $A$ plays the following strategy for any consumer $x$ : with probability $\Delta_{A}$, it draws $p_{a}(x)$ from some distribution $G_{A}^{x}(p)$ that is defined on the interval $\left[(1-\beta) p_{B}^{x}, p_{B}^{x}\right]$, while with probability $1-\Delta_{A}$, it sets $p_{a}(x)=p_{B}^{x}$. As $G_{A}^{x}\left((1-\beta) p_{B}^{x}\right)=0$, firm $B$ 's expected profit from playing $p_{B}(x)=(1-\beta) p_{B}^{x}$ is equal to

$$
\tilde{\pi}_{B}\left((1-\beta) p_{B}^{x}\right)=\Delta_{A}\left(1-G_{A}^{x}\left((1-\beta) p_{B}^{x}\right)\right)(1-\beta) p_{B}^{x}+\left(1-\Delta_{A}\right)(1-\beta) p_{B}^{x}=(1-\beta) p_{B}^{x} .
$$


By taking limits, we obtain that firm $B$ 's expected profit from playing $p_{B}(x)=p_{B}^{x}$ is given by

$$
\lim _{p \rightarrow p_{B}^{x}} \tilde{\pi}_{B}(p)=\lim _{p \rightarrow p_{B}^{x}}\left(\Delta_{A}\left(1-G_{A}^{x}(p)\right) p+\left(1-\Delta_{A}\right) p\right)=\left(1-\Delta_{A}\right) p_{B}^{x} .
$$

For firm $B$ to be indifferent between playing $p_{B}(x)=(1-\beta) p_{B}^{x}$ or $p_{B}(x)=p_{B}^{x}$, firm $A$ must choose $\Delta_{A}$ such that $(1-\beta) p_{B}^{x}=\left(1-\Delta_{A}\right) p_{B}^{x}$, or $\Delta_{A}=\beta$. In addition, firm $B$ must also be indifferent between any $p \in\left[(1-\beta) p_{B}^{x}, p_{B}^{x}\right]$, which requires $(1-\beta) p+\beta\left(1-G_{A}^{x}(p)\right) p=$ $(1-\beta) p_{B}^{x}$, which is equivalent to

$$
G_{A}^{x}(p)=\frac{p-(1-\beta) p_{B}^{x}}{\beta p}=G^{x}(p)
$$

Given that $p_{B}>0$, it follows that for any $x$ such that $r(x)>0$, both firms make positive expected profits equal to $(1-\beta) p_{B}^{x}=(1-\beta) \min \left\{p_{B}, r(x)\right\}$.

Stage 1. In any mixed strategy equilibrium, firm $B$ must be indifferent between charging any price $p$ in the support of $H_{B}, p_{l}, \lim _{p \rightarrow p^{m}}$, and $\bar{r}$. Recall that by choosing $\bar{r}$, firm $B$ can secure a profit of $\pi_{B}^{\min }=\beta(1-\beta) \lambda \hat{r}$. If firm $B$ chooses some price $p$, then with probability $\beta \lambda$, firm $B$ competes for consumers on the transparent segment and will obtain expected profits of $\stackrel{\circ}{\pi}_{B}(p)$; with probability $1-\beta \lambda$, firm $B$ does not recognize consumers and will only obtain positive profits if firm $A$ does not recognize consumers either (the segment is opaque), which happens with probability $(1-\lambda) /(1-\beta \lambda)$ (conditional on the event in which firm $B$ does not recognize a consumer). Therefore, the probability that firm $B$ is active on the opaque segment is $(1-\lambda)$. Given that with probability $\nabla_{A}$, firm $A$ draws $p_{A}$ from the distribution $H_{A}(p)$ defined on $\left[p_{l}, p^{m}\right]$ and, with probability $1-\nabla_{A}$, sets $p_{A}=p^{m}$, we can compute firm $B$ 's expected profit from choosing price $p$ as

$$
\Pi_{B}^{e}(p)=\beta \lambda \stackrel{\circ}{\pi}_{B}(p)+(1-\lambda) p(1-F(p))\left[\left(1-\nabla_{A}\right)+\nabla_{A}\left(1-H_{A}(p)\right)\right] .
$$

As firm $B$ must be indifferent between charging any price $p$ in the support of $H_{B}$ and $\bar{r}$, we must have that $\Pi_{B}^{e}(p)=\pi_{B}^{\mathrm{min}}$, from which we obtain

$$
\begin{aligned}
H_{A}(p) & =\frac{\beta \lambda\left(\stackrel{\circ}{\pi}_{B}(p)-(1-\beta) \hat{r}\right)+(1-\lambda) p(1-F(p))}{(1-\lambda) p(1-F(p)) \nabla_{A}} \\
& =1-\frac{\beta \lambda\left((1-\beta) \hat{r}-\stackrel{\circ}{\pi}_{B}(p)\right)-(1-\lambda)\left(1-\nabla_{A}\right) p(1-F(p))}{(1-\lambda) p(1-F(p)) \nabla_{A}} .
\end{aligned}
$$

As $H_{A}\left(p_{l}\right)=0$, firm $B$ achieves the following expected profit when charging $p_{l}$ :

$$
\begin{aligned}
\Pi_{B}^{e}\left(p_{l}\right) & =\beta \lambda \int_{0}^{p_{l}}(1-\beta) r f(r) d r+\beta \lambda \int_{p_{l}}^{\bar{r}}(1-\beta) p_{l} f(r) d r+(1-\lambda) p_{l}\left(1-F\left(p_{l}\right)\right) \\
& =\beta \lambda \int_{0}^{p_{l}}(1-\beta) r f(r) d r+(\beta \lambda(1-\beta)+1-\lambda) p_{l}\left(1-F\left(p_{l}\right)\right)
\end{aligned}
$$

As firm $B$ must be indifferent between charging $p_{l}$ and $\bar{r}$, we have that $\Pi_{B}^{e}\left(p_{l}\right)=\beta \lambda(1-\beta) \hat{r}$, which implicitly defines $p_{l}$ as a function of $\beta, \lambda$ and $F$ :

$$
p_{l}=\frac{\beta \lambda(1-\beta)\left(\hat{r}-\int_{0}^{p_{l}} r f(r) d r\right)}{(\beta \lambda(1-\beta)+1-\lambda)\left(1-F\left(p_{l}\right)\right)} .
$$


As $H_{A}\left(p^{m}\right)=1$, firm $B$ 's expected profit from charging $p^{m}$ is obtained as follows

$$
\Pi_{B}^{e}\left(p^{m}\right)=\beta \lambda \stackrel{\circ}{\pi}_{B}\left(p^{m}\right)+(1-\lambda) p^{m}\left(1-F\left(p^{m}\right)\right)\left(1-\nabla_{A}\right),
$$

which must be equal to $\pi_{B}^{\min }=\beta(1-\beta) \lambda \hat{r}$. We obtain thus

$$
1-\nabla_{A}=\frac{\beta \lambda(1-\beta)\left(\hat{r}-\int_{0}^{p^{m}} r f(r) d r-\pi^{m}\right)}{(1-\lambda) \pi^{m}}>0 .
$$

It must also be that $\nabla_{A}>0$. This is satisfied when

$$
(1-\lambda) \pi^{m} \geq \beta \lambda(1-\beta)\left(\hat{r}-\int_{0}^{p^{m}} r f(r) d r-\pi^{m}\right) .
$$

When this condition is not satisfied, then $\nabla_{A}=0$ and firm $A$ plays $p_{A}=p^{m}$ with probability 1 . In this case, firm $B$ 's optimal response is to play $p_{B}=\bar{r}$ with probability 1. In other words, we obtain an equilibrium in pure strategies in which firm $A$ will be a monopolist in markets for consumers which are not identified, while firm $B$ gives up any sales to consumers it does not identify and chooses a price that maximizes its expected profits in the transparent segment.

Now assume that $\nabla_{A}>0$, it must be that firm $A$ is indifferent between charging $p^{m}$ and $p_{l}$. Denote by $\hat{\pi}_{A}\left(\nabla_{B}, H_{B}\right)$ the expected profit of firm $A$ given firm $B$ 's regular price strategy. We then have:

$$
\Pi_{A}^{e}(p)=\lambda \hat{\pi}_{A}\left(\nabla_{B}, H_{B}\right)+(1-\lambda) p(1-F(p))\left(1-\nabla_{B}+\nabla_{B}\left(1-H_{B}(p)\right)\right) .
$$

As $H_{B}\left(p^{m}\right)=1$ and $H_{B}\left(p_{l}\right)=0$, it follows that

$$
\begin{aligned}
\Pi_{A}^{e}\left(p^{m}\right) & =\lambda \hat{\pi}_{A}\left(\nabla_{B}, H_{B}\right)+(1-\lambda) p^{m}\left(1-F\left(p^{m}\right)\right)\left(1-\nabla_{B}\right), \\
\Pi_{A}^{e}\left(p_{l}\right) & =\lambda \hat{\pi}_{A}\left(\nabla_{B}, H_{B}\right)+(1-\lambda) p_{l}\left(1-F\left(p_{l}\right)\right) .
\end{aligned}
$$

For firm $A$ to be indifferent, we need $\Pi_{A}^{e}\left(p^{m}\right)=\Pi_{A}^{e}\left(p_{l}\right)$, which is equivalent to

$$
1-\nabla_{B}=\frac{p_{l}\left(1-F\left(p_{l}\right)\right)}{p^{m}\left(1-F\left(p^{m}\right)\right)}=\frac{\pi_{l}}{\pi^{m}} .
$$

Since $\pi_{l} \leq \pi^{m}$, it must be that $\nabla_{B} \in[0,1]$. It must also be the case that $\Pi_{A}^{e}(p)=\Pi_{A}^{e}\left(p_{l}\right)$, from which we obtain

$$
\begin{aligned}
H_{B}(p) & =1-\frac{\pi_{l}-p(1-F(p))\left(1-\nabla_{B}\right)}{p(1-F(p)) \nabla_{B}}=1-\frac{\pi_{l}-p(1-F(p)) \frac{\pi_{l}}{\pi^{m}}}{p(1-F(p))\left(1-\frac{\pi_{l}}{\pi^{m}}\right)} \\
& =1-\frac{\pi_{l}}{\pi^{m}-\pi_{l}}\left(\frac{\pi^{m}}{p(1-F(p))}-1\right) .
\end{aligned}
$$

This completes the proof.

\subsubsection{Comparative statics results}

\section{Proof of Corollary 1 .}


1. Let us show that $\frac{\partial p_{l}}{\partial \lambda}>0$. We have that

$$
p_{l}(\beta \lambda(1-\beta)+1-\lambda)\left(1-F\left(p_{l}\right)\right)=\beta \lambda(1-\beta)\left(\hat{r}-\int_{0}^{p_{l}} r f(r) d r\right) .
$$

Totally differentiating with respect to $\lambda$ we obtain

$$
\begin{aligned}
& \frac{\partial p_{l}}{\partial \lambda}\left[(\beta \lambda(1-\beta)+1-\lambda)\left(1-F\left(p_{l}\right)-p_{l} f\left(p_{l}\right)\right)\right]+p_{l}(\beta(1-\beta)-1)\left(1-F\left(p_{l}\right)\right) \\
= & \beta(1-\beta)\left(\hat{r}-\int_{0}^{p_{l}} r f(r) d r\right)-\frac{\partial p_{l}}{\partial \lambda} \beta \lambda(1-\beta) \int_{0}^{p_{l}} r f(r) d r .
\end{aligned}
$$

or

$$
\begin{aligned}
\frac{\partial p_{l}}{\partial \lambda} \underbrace{\left[(\beta \lambda(1-\beta)+1-\lambda)\left(1-F\left(p_{l}\right)-p_{l} f\left(p_{l}\right)\right)+\beta \lambda(1-\beta) \int_{0}^{p_{l}} r f(r) d r\right]}_{=A} & (\underbrace{\hat{r}-\int_{0}^{p_{l}} r f(r) d r-p_{l}\left(1-F\left(p_{l}\right)\right)}_{=B})+p_{l}\left(1-F\left(p_{l}\right)\right) .
\end{aligned}
$$

Since $p_{l}<p^{m}$ we have that $1-F\left(p_{l}\right)-p_{l} f\left(p_{l}\right)>1-F\left(p^{m}\right)-p^{m} f\left(p^{m}\right)=\left.\frac{\partial \pi}{\partial p}\right|_{p=p^{m}}=$ 0 , from which we obtain that $A>0$. Also note that $(1-\beta) \hat{r}=\stackrel{\circ}{\pi}_{B}(\bar{r})>\stackrel{\circ}{\pi}_{B}\left(p_{l}\right)=$ $(1-\beta)\left(\int_{0}^{p_{l}} r f(r) d r+p_{l}\left(1-F\left(p_{l}\right)\right)\right)$ and hence $B>0$. From this, it follows that $\frac{\partial p_{l}}{\partial \lambda}>0$.

2. Let us show that $\frac{\partial \nabla_{A}}{\partial \lambda}<0$. Observe that

$$
\nabla_{A}=1-\frac{\beta(1-\beta)\left(\hat{r}-\int_{0}^{p^{m}} r f(r) d r-\pi^{m}\right)}{\pi^{m}} \times \frac{\lambda}{1-\lambda},
$$

and hence

$$
\frac{\partial \nabla_{A}}{\partial \lambda}=-\frac{\beta(1-\beta)\left(\hat{r}-\int_{0}^{p^{m}} r f(r) d r-\pi^{m}\right)}{\pi^{m}} \times \frac{1}{(1-\lambda)^{2}}<0,
$$

which can be rewritten as:

$$
\frac{\partial \nabla_{A}}{\partial \lambda}=-\eta_{A} \times \frac{1}{\lambda(1-\lambda)}<0 .
$$

3. Let us show that $\frac{\partial H_{A}(p)}{\partial \lambda}>0$. Recall that:

$$
\begin{gathered}
H_{A}(p)=1-\frac{\beta(1-\beta)\left(\hat{r}-\int_{0}^{p} r f(r) d r-p(1-F(p))\right)}{p(1-F(p))} \times \frac{\lambda}{(1-\lambda) \nabla_{A}}+\frac{1-\nabla_{A}}{\nabla_{A}} ; \\
\frac{\partial H_{A}(p)}{\partial \lambda}=-\frac{\beta(1-\beta)\left(\hat{r}-\int_{0}^{p} r f(r) d r-p(1-F(p))\right)}{p(1-F(p))} \times \frac{(1-\lambda) \nabla_{A}-\lambda\left(\frac{\partial \nabla_{A}}{\partial \lambda}(1-\lambda)-\nabla_{A}\right)}{\left((1-\lambda) \nabla_{A}\right)^{2}} \\
+\frac{-\frac{\partial \nabla_{A}}{\partial \lambda} \nabla_{A}-\frac{\partial \nabla_{A}}{\partial \lambda}\left(1-\nabla_{A}\right)}{\left(\nabla_{A}\right)^{2}}
\end{gathered}
$$


Grouping terms, we obtain

$\frac{\partial H_{A}(p)}{\partial \lambda}=-\frac{\beta(1-\beta)\left(\hat{r}-\int_{0}^{p} r f(r) d r-p(1-F(p))\right)}{p(1-F(p))} \times \frac{\nabla_{A}-\frac{\partial \nabla_{A}}{\partial \lambda} \lambda(1-\lambda)}{\left((1-\lambda) \nabla_{A}\right)^{2}}-\frac{\frac{\partial \nabla_{A}}{\partial \lambda}}{\left(\nabla_{A}\right)^{2}}$.

Note that $\nabla_{A}-\frac{\partial \nabla_{A}}{\partial \lambda} \lambda(1-\lambda)=1-\eta_{A}+\eta_{A}=1$ and hence we have

$\frac{\partial H_{A}(p)}{\partial \lambda}=-\frac{\beta(1-\beta)\left(\hat{r}-\int_{0}^{p} r f(r) d r-p(1-F(p))\right)}{p(1-F(p))} \times \frac{1}{\left((1-\lambda) \nabla_{A}\right)^{2}}+\frac{\eta_{A}}{\lambda(1-\lambda)\left(\nabla_{A}\right)^{2}}$.

Since

$$
\begin{gathered}
\frac{\beta(1-\beta)\left(\hat{r}-\int_{0}^{p} r f(r) d r-p(1-F(p))\right)}{p(1-F(p))} \\
=\frac{\beta(1-\beta) \lambda\left(\hat{r}-\int_{0}^{p} r f(r) d r-p(1-F(p))\right)}{(1-\lambda) p(1-F(p))} \times \frac{1-\lambda}{\lambda}>\eta_{A} \frac{1-\lambda}{\lambda},
\end{gathered}
$$

we have that

$$
\begin{gathered}
\frac{\partial H_{A}(p)}{\partial \lambda}<-\eta_{A} \frac{1-\lambda}{\lambda} \times \frac{1}{\left((1-\lambda) \nabla_{A}\right)^{2}}+\frac{\eta_{A}}{\lambda(1-\lambda)\left(\nabla_{A}\right)^{2}} \\
\quad \text { or } \frac{\partial H_{A}(p)}{\partial \lambda}<-\frac{\eta_{A}}{\lambda(1-\lambda)\left(\nabla_{A}\right)^{2}}+\frac{\eta_{A}}{\lambda(1-\lambda)\left(\nabla_{A}\right)^{2}}=0 .
\end{gathered}
$$

4. Let us show that $\frac{\partial \nabla_{B}}{\partial \lambda}<0$. Recall that $\nabla_{B}=1-\frac{p_{l}\left(1-F\left(p_{l}\right)\right.}{\pi^{m}}$. Since $\frac{\partial p_{l}}{\partial \lambda}>0$ and $p_{l}<p^{m}$, we have that:

$$
\frac{\partial \nabla_{B}}{\partial \lambda}=-\frac{\frac{\partial p_{l}}{\partial \lambda}\left(1-F\left(p_{l}\right)-p_{l} f\left(p_{l}\right)\right)}{\pi^{m}}<0
$$

5. Let us show that $\frac{\partial H_{B}(p)}{\partial \lambda}<0$. Recalling that

$$
H_{B}(p)=1-\frac{1-\nabla_{B}}{\nabla_{B}} \times\left(\frac{\pi^{m}}{p(1-F(p))}-1\right)
$$

we observe that

$$
\frac{\partial H_{B}(p)}{\partial \lambda}=\left(\frac{\pi^{m}}{p(1-F(p))}-1\right) \frac{\frac{\partial \nabla_{B}(p)}{\partial \lambda}}{\left(\nabla_{B}\right)^{2}}
$$

and hence $\frac{\partial H_{B}(p)}{\partial \lambda}<0$ since $\frac{\partial \nabla_{B}(p)}{\partial \lambda}<0$.

Proof of Corollary 2, We demonstrate here that for high levels of asymmetry $\left(\beta<\frac{1}{2}\right)$, increasing $\beta$ increases the uniform prices, whereas the opposite is true when the level of symmetry is high $\left(\beta>\frac{1}{2}\right)$. That is, we show

$$
\frac{\partial p_{l}}{\partial \beta} \lesseqgtr 0 \Leftrightarrow \beta \gtreqless \frac{1}{2} \text { and } \frac{\partial \nabla_{A}}{\partial \beta}, \frac{\partial \nabla_{B}}{\partial \beta}, \frac{\partial H_{A}(p)}{\partial \beta} \frac{\partial H_{B}(p)}{\partial \beta} \lesseqgtr 0 \Leftrightarrow \beta \lesseqgtr \frac{1}{2} \text {. }
$$


1. Let us show that $\frac{\partial p_{l}}{\partial \beta}>0 \Longleftrightarrow \beta<\frac{1}{2}$. We have that

$$
p_{l}(\beta \lambda(1-\beta)+1-\lambda)\left(1-F\left(p_{l}\right)\right)=\beta \lambda(1-\beta)\left(\hat{r}-\int_{0}^{p_{l}} r f(r) d r\right) .
$$

Totally differentiating with respect to $\beta$ we obtain

$$
\begin{aligned}
& \frac{\partial p_{l}}{\partial \beta}\left[(\beta \lambda(1-\beta)+1-\lambda)\left(1-F\left(p_{l}\right)-p_{l} f\left(p_{l}\right)\right)\right]+p_{l} \lambda(1-2 \beta)\left(1-F\left(p_{l}\right)\right) \\
= & \lambda(1-2 \beta)\left(\hat{r}-\int_{0}^{p_{l}} r f(r) d r\right)-\frac{\partial p_{l}}{\partial \beta} \beta \lambda(1-\beta) \int_{0}^{p_{l}} r f(r) d r .
\end{aligned}
$$

or

$$
\begin{gathered}
\frac{\partial p_{l}}{\partial \lambda} \underbrace{\left[(\beta \lambda(1-\beta)+1-\lambda)\left(1-F\left(p_{l}\right)-p_{l} f\left(p_{l}\right)\right)+\beta \lambda(1-\beta) \int_{0}^{p_{l}} r f(r) d r\right]}_{=A} \\
=\lambda(1-2 \beta)(\underbrace{\hat{r}-\int_{0}^{p_{l}} r f(r) d r-p_{l}\left(1-F\left(p_{l}\right)\right)}_{=B}) .
\end{gathered}
$$

Since $p_{l}<p^{m}$ we have that $1-F\left(p_{l}\right)-p_{l} f\left(p_{l}\right)>1-F\left(p^{m}\right)-p^{m} f\left(p^{m}\right)=\left.\frac{\partial \pi}{\partial p}\right|_{p=p^{m}}=$ 0 , from which we obtain that $A>0$. Also note that $\left.(1-\beta) \hat{r}=\stackrel{\circ}{\pi}_{B}(\bar{r})>\stackrel{\circ}{\pi}_{B}\left(p_{l}\right)\right)=$ $(1-\beta)\left(\int_{0}^{p_{l}} r f(r) d r+p_{l}\left(1-F\left(p_{l}\right)\right)\right)$ and hence $B>0$. From this, it follows that $\frac{\partial p_{l}}{\partial \beta} \lesseqgtr 0 \Longleftrightarrow \beta \gtreqless \frac{1}{2}$.

2. Let us evaluate $\frac{\partial \nabla_{A}}{\partial \beta}$. Recall that

$$
\begin{gathered}
\nabla_{A}=1-\beta(1-\beta)\left(\frac{\lambda}{(1-\lambda) \pi^{m}}\left(\hat{r}-\int_{0}^{p^{m}} r f(r) d r-\pi^{m}\right)\right), \text { so that } \\
\frac{\partial \nabla_{A}}{\partial \beta}=-(1-2 \beta)\left(\frac{\lambda}{(1-\lambda) \pi^{m}}\left(\hat{r}-\int_{0}^{p^{m}} r f(r) d r-\pi^{m}\right)\right)
\end{gathered}
$$

We thus have that

$$
\frac{\partial \nabla_{A}}{\partial \beta}=-\frac{\partial \eta_{A}}{\partial \beta}=-\eta_{A} \times \frac{1-2 \beta}{\beta(1-\beta)} \lesseqgtr 0 \Longleftrightarrow \beta \lesseqgtr \frac{1}{2} .
$$

3. We now turn to $\frac{\partial H_{A}(p)}{\partial \beta}$. Recall that:

$$
H_{A}(p)=1-\frac{\beta(1-\beta)}{\nabla_{A}} \frac{\lambda\left(\hat{r}-\int_{0}^{p} r f(r) d r-p(1-F(p))\right)}{(1-\lambda) p(1-F(p))}+\frac{1-\nabla_{A}}{\nabla_{A}}, \text { so that }
$$




$$
\begin{aligned}
\frac{\partial H_{A}(p)}{\partial \lambda}= & -\frac{\lambda\left(\hat{r}-\int_{0}^{p} r f(r) d r-p(1-F(p))\right)}{(1-\lambda) p(1-F(p))} \times \frac{(1-2 \beta) \nabla_{A}-\left(\frac{\partial \nabla_{A}}{\partial \beta} \beta(1-\beta)\right)}{\left(\nabla_{A}\right)^{2}}-\frac{\frac{\partial \nabla_{A}}{\partial \beta}}{\left(\nabla_{A}\right)^{2}} \\
= & -\frac{\lambda\left(\hat{r}-\int_{0}^{p} r f(r) d r-p(1-F(p))\right)}{(1-\lambda) p(1-F(p))} \times \frac{(1-2 \beta) \nabla_{A}+\left(1-\nabla_{A}\right)(1-2 \beta)}{\left(\nabla_{A}\right)^{2}} \\
& +\frac{\left(1-\nabla_{A}\right)(1-2 \beta)}{\beta(1-\beta)\left(\nabla_{A}\right)^{2}} \\
= & \frac{1-2 \beta}{\left(\nabla_{A}\right)^{2}}[\underbrace{\frac{1-\nabla_{A}}{\beta(1-\beta)}-\frac{\lambda\left(\hat{r}-\int_{0}^{p} r f(r) d r-p(1-F(p))\right)}{(1-\lambda) p(1-F(p))}}_{C}] .
\end{aligned}
$$

Note that, since $p \leq p^{m}$, we have

$$
\begin{gathered}
\frac{\lambda\left(\hat{r}-\int_{0}^{p} r f(r) d r-p(1-F(p))\right)}{(1-\lambda) p(1-F(p))} \geq \\
\frac{\lambda\left(\hat{r}-\int_{0}^{p^{m}} r f(r) d r-p^{m}\left(1-F\left(p^{m}\right)\right)\right)}{(1-\lambda) p^{m}\left(1-F\left(p^{m}\right)\right)}=\frac{1-\nabla_{A}}{\beta(1-\beta)} .
\end{gathered}
$$

Hence we obtain that $C \leq 0$, which implies that $\frac{\partial H_{A}(p)}{\partial \beta} \lesseqgtr 0 \Longleftrightarrow \beta \lesseqgtr \frac{1}{2}$. In other words, when $\beta$ is low, increasing $\beta$ increases prices on the opaque segment, as firms avoid cut-throat competition. In other words, when $\beta$ is relatively high, increasing $\beta$ make the firms even more symmetric, thereby increasing competition and lowering prices.

4. Now we consider $\frac{\partial \nabla_{B}}{\partial \beta}$. Recall that $\nabla_{B}=1-\frac{p_{l}\left(1-F\left(p_{l}\right)\right.}{\pi^{m}}$. We then obtain, since $\frac{\partial p_{l}}{\partial \beta}>0$ and $p_{l}<p^{m}$, that:

$$
\frac{\partial \nabla_{B}}{\partial \beta}=-\frac{\frac{\partial p_{l}}{\partial \beta}\left(1-F\left(p_{l}\right)-p_{l} f\left(p_{l}\right)\right)}{\pi^{m}} \lesseqgtr 0 \Longleftrightarrow \beta \lesseqgtr \frac{1}{2} .
$$

5. We finally discuss the sign of $\frac{\partial H_{B}(p)}{\partial \beta}$. As

$$
H_{B}(p)=1-\frac{1-\nabla_{B}}{\nabla_{B}} \times\left(\frac{\pi^{m}}{p(1-F(p))}-1\right),
$$

we observe that

$$
\frac{\partial H_{B}(p)}{\partial \beta}=\left(\frac{\pi^{m}}{p(1-F(p))}-1\right) \frac{\frac{\partial \nabla_{B}(p)}{\partial \beta}}{\left(\nabla_{B}\right)^{2}},
$$

and hence

$$
\frac{\partial H_{B}(p)}{\partial \beta} \lesseqgtr 0 \Longleftrightarrow \beta \lesseqgtr \frac{1}{2} \text {. }
$$


Proof of Corollary 3. Let us consider $\frac{\partial G^{x}(p)}{\partial \beta}$ for $p \in\left[(1-\beta) p_{B}^{x}, p_{B}^{x}\right]$.

$$
\frac{\partial G^{x}(p)}{\partial \beta}=\frac{\partial}{\partial \beta}\left(\frac{1}{\beta}-\frac{p_{B}^{x}}{p} \frac{1-\beta}{\beta}\right)=\frac{p_{B}^{x}-p}{\beta^{2} p}>0 \text { for any } p<p_{B}^{x} .
$$

Profit ranking. We establish here that the better-informed firm (firm A) earns higher expected profits than the less-informed firm (firm B).

We know that firms are indifferent between all equilibrium uniform prices. Hence, the expected profit from any uniform price is equal to the expected profit from playing the lowest possible uniform price $p_{l}$. When setting their price equal to $p_{l}$, both firms have the same expected profit from the opaque segment. Moreover, firm $A$ gets additional profits from the translucent segment since she has a lower targeted price than firm $B$ 's observed uniform price. Finally, the expected profit from the transparent market is higher for firm $A$ than for firm $B$, since firm $B$ minimizes profits from the transparent market by setting $p_{B}=p_{l}$. Firm $A$ 's expected profits on the transparent market are averaged over all possible uniform prices firm $B$ may choose, either between $p_{l}$ and $p^{m}$ or equal to $\bar{r}$, and these expected profits do not depend on its own uniform price, $p_{A}$. Adding these three effects, we see that $A$ enjoys higher expected profits.

\subsubsection{Uniform distribution}

Suppose that consumer valuations are uniformly distributed on the unit interval and, to ease the exposition, define $k \equiv \beta(1-\beta)$. We know from Proposition 3 that the mixedstrategy equilibrium in uniform prices is as follows: with probability $1-\nabla_{A}$, firm $A$ plays $p_{A}=p^{m}$ and with probability $\nabla_{A}$, it draws a price from the distribution $H_{A}(p)$ defined on $\left[p_{l}, p^{m}\right)$; with probability $1-\nabla_{B}$, firm $B$ plays $p_{B}=\bar{r}=1$ and with probability $\nabla_{B}$, it draws a price from the distribution $H_{B}(p)$ defined on $\left[p_{l}, p^{m}\right)$. Then, supposing that $2(1-\lambda)>\lambda k$, we find the following results ${ }^{32}$

$$
\begin{aligned}
\nabla_{A} & =\frac{2(1-\lambda)-\lambda k}{2(1-\lambda)}, \nabla_{B}=\left(\frac{2(1-\lambda)-\lambda k}{2(1-\lambda)+\lambda k}\right)^{2}, \\
H_{A}(p) & =\frac{1}{p} \frac{(2(1-\lambda)+\lambda k) p-\lambda k}{2(1-\lambda)-\lambda k} \\
H_{B}(p) & =\frac{(2(1-\lambda)(1-p)-\lambda k p)(2(1-\lambda) p-\lambda k(1-p))}{p(1-p)(2(1-\lambda)-\lambda k)^{2}}, \\
p_{l} & =\frac{\lambda k}{2(1-\lambda)+\lambda k}, p^{m}=\frac{1}{2} .
\end{aligned}
$$

It is readily checked that $\nabla_{A}, \nabla_{B}, H_{A}(p)$, and $H_{B}(p)$ are decreasing function of $\lambda$ and $k$, while $p_{l}$ is an increasing function of $\lambda$ and $k$. This means first, as stated in Corollary 1. that the distributions of uniform prices are pushed to the right for both firms when profiling abilities improve. Second, recalling that $k \equiv \beta(1-\beta)$ is bell-shaped in $\beta$ with a maximum at $\beta=1 / 2$, we also have that the distribution of uniform prices of both firms

\footnotetext{
${ }^{32}$ If $2(1-\lambda) \leq \lambda k$, then $\nabla_{A}=\nabla_{B}=0$, meaning that, with probability 1 , firm $A$ sets $p_{A}=1 / 2$ and firm $B$ sets $p_{B}=1$. See Appendix 7.2 .2 for the detailed computations.
} 
Figure 5: Impact of improved profiling on price distributions

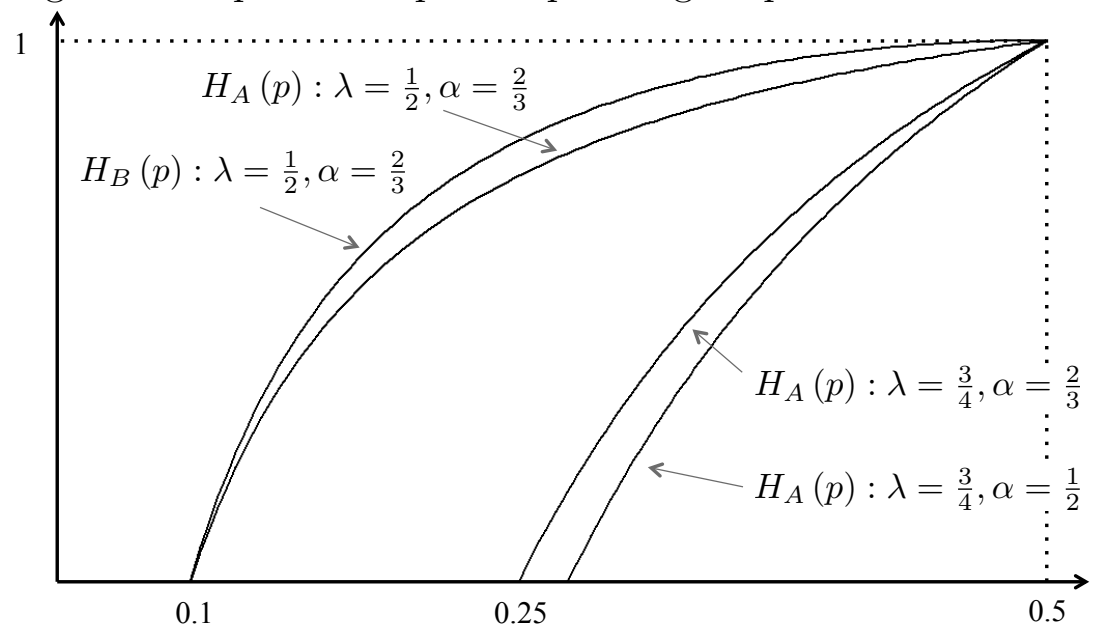

are also pushed to the right when $\beta$ comes closer to $1 / 2$, as indicated in Corollary 2 . Third, we observe in this example that $H_{B}(p)>H_{A}(p)$ for all $p$, meaning that firm $B$ always puts more weight on lower prices than firm $A$ whenever both firms randomize. Figure 5 illustrates these results by representing $H_{A}(p)$ and $H_{B}(p)$ for different values of $\lambda$ and $\beta$ : going from left to right, the first locus is $H_{B}(p)$ for $(\lambda, \beta)=\left(\frac{1}{2}, \frac{2}{3}\right)$, the second is $H_{A}(p)$ for $(\lambda, \beta)=\left(\frac{1}{2}, \frac{2}{3}\right)$, the third is $H_{A}(p)$ for $(\lambda, \beta)=\left(\frac{3}{4}, \frac{2}{3}\right)$, and the fourth is $H_{A}(p)$ for $(\lambda, \beta)=\left(\frac{3}{4}, \frac{1}{2}\right)$.

Let $F($.$) be the uniform distribution on [0,1]$. It follows that the average valuation $(\hat{r})$ and the monopoly price $\left(p^{m}\right)$ are both equal to $1 / 2$; the monopoly profit is computed as $\pi^{m}=1 / 4$. Furthermore, we have

$$
\stackrel{\circ}{\pi}_{B}(p)=\int_{0}^{p}(1-\beta) r f(r) d r+(1-\beta) p(1-F(p))=(1-\beta)\left(p-\frac{1}{2} p^{2}\right),
$$

and $\pi_{B}^{m i n}=\frac{1}{2} \lambda \beta(1-\beta)$. The lower bound of uniform price $p_{l}$ is then defined by

$$
p_{l}=\frac{\beta \lambda(1-\beta)\left(\hat{r}-\int_{0}^{p_{l}} r f(r) d r\right)}{(\beta \lambda(1-\beta)+1-\lambda)\left(1-F\left(p_{l}\right)\right)} \Leftrightarrow p_{l}=\frac{\beta \lambda(1-\beta)}{2(1-\lambda)+\beta \lambda(1-\beta)} .
$$

We observe that $p_{l}$ is increasing in $\lambda$, increasing in $\beta$ if $\beta \leq 1 / 2$, and decreasing in $\beta$ if $\beta>1 / 2$. This implies that the uniform price is less dispersed as tracking technologies become more precise and the difference in profiling technologies between the two firms are neither too large nor too small (i.e., $p_{l}$ is at the highest level when $\beta=1 / 2$ ).

The probability of firm $A$ randomizing its uniform price is defined by

$$
\nabla_{A}=1-\frac{\beta \lambda(1-\beta)}{(1-\lambda) \pi^{m}}\left(\hat{r}-\int_{0}^{p^{m}} r f(r) d r-\pi^{m}\right)=1-\frac{\beta \lambda(1-\beta)}{2(1-\lambda)} .
$$

This probability is decreasing in $\lambda$, decreasing in $\beta$ if $\beta \leq 1 / 2$, and increasing in $\beta$ if $\beta>1 / 2$. 
When $2(1-\lambda) \leq \beta \lambda(1-\beta)$, we have $\nabla_{A}=0$, so that firm $A$ plays $p_{A}=p^{m}=1 / 2$ with probability 1 , whereas firm $B$ plays $p_{B}=1$ with probability 1 . When $2(1-\lambda)>\beta \lambda(1-\beta)$, we have $\nabla_{A}>0$; the probability of firm $\mathrm{B}$ randomizing its uniform is then defined by

$$
\nabla_{B}=1-\frac{p_{l}\left(1-F\left(p_{l}\right)\right)}{p^{m}\left(1-F\left(p^{m}\right)\right)}=\left(\frac{2(1-\lambda)-\beta \lambda(1-\beta)}{2(1-\lambda)+\beta \lambda(1-\beta)}\right)^{2} .
$$

The latter probability is decreasing in $\lambda$, decreasing in $\beta$ if $\beta \leq 1 / 2$, and increasing in $\beta$ if $\beta>1 / 2$.

Thus, firm B plays $p_{B}=1$ with probability $1-\nabla_{B}$, and draws a price from $\left[p_{l}, 1 / 2\right]$ with probability $\nabla_{B}$ and with a distribution

$$
H_{B}(p)=1-\frac{1-\nabla_{B}}{\nabla_{B}}\left(\frac{1}{4 p(1-p)}-1\right),
$$

which is increasing in $\nabla_{B}$.

Firm A plays $p_{A}=1 / 2$ with probability $1-\nabla_{A}$, and draws a price from $\left[p_{l}, 1 / 2\right]$ with probability $\nabla_{A}$ and with a distribution

$$
H_{A}(p)=\frac{1}{\nabla_{A}}\left(1-\frac{\lambda \beta(1-\beta)(1-p)}{2(1-\lambda) p}\right) .
$$

Effect of improved profiling on consumer surplus. Suppose that $\beta=1 / 2$ and $\lambda>8 / 9$, which implies that $2(1-\lambda)-\beta \lambda(1-\beta)<0$, so that $\nabla_{A}=0$. Under these conditions, firm $A$ plays $p_{A}=p^{m}=1 / 2$ with probability 1 , whereas firm B plays $p_{B}=1$ with probability 1 . Consumers thus face the monopoly price when they remain anonymous. For the consumers whom they profile, both firms draw their personalized price from the interval $[r / 2, r]$ according to the distribution function $G(p)=2-(r / p)$, with density $g(p)=r / p^{2}$. Hence, when consumers are profiled only by firm $A$, they face one draw from $G(p)$. When they are profiled by both firms, they face the minimum of two draws from $G(p)$, which follows the distribution $\Gamma(p)=G(p)^{2}$, with density $\gamma(p)=2 G(p) g(p)$.

Suppose that $\beta=1 / 2$ and $\lambda>8 / 9$, which implies that $2(1-\lambda)-\beta \lambda(1-\beta)<0$, so that $\nabla_{A}=0$. Under these conditions, firm $A$ plays $p_{A}=p^{m}=1 / 2$ with probability 1 , whereas firm B plays $p_{B}=1$ with probability 1 . Consumer surplus is then computed as:

$$
\begin{aligned}
\left.C S(\lambda)\right|_{\lambda \geq 8 / 9}= & (1-\lambda) \int_{\frac{1}{2}}^{1}\left(r-\frac{1}{2}\right) d r+\frac{\lambda}{2} \int_{0}^{1}\left(r-\int_{\frac{r}{2}}^{r} p g(p) d p\right) d r \\
& +\frac{\lambda}{2} \int_{0}^{1}\left(r-\int_{\frac{r}{2}}^{r} p \gamma(p) d p\right) d r=(1-\lambda) \frac{1}{8}+\lambda \frac{4-5 \ln 2}{4} .
\end{aligned}
$$

It follows that

$$
\left.\frac{\partial C S(\lambda)}{\partial \lambda}\right|_{\lambda \geq 8 / 9}=-\frac{1}{8}+\frac{4-5 \ln 2}{4}=\frac{7-10 \ln 2}{8} \approx 0,0086>0 .
$$

For any $\lambda>8 / 9$, firm $A$ 's expected profit is computed as:

$$
\begin{aligned}
\left.\Pi_{A}(\lambda)\right|_{\lambda \geq 8 / 9} & =(1-\lambda) \int_{\frac{1}{2}}^{1} \frac{1}{2} d r+\lambda\left(\frac{1}{2} \int_{0}^{1} \int_{\frac{r}{2}}^{r} p g(p) d p d r+\frac{1}{2} \int_{0}^{1} \int_{\frac{r}{2}}^{r} p(1-G(p)) g(p) d p d r\right) \\
& =(1-\lambda) \frac{1}{4}+\lambda \frac{1}{2} \int_{0}^{1} \int_{\frac{r}{2}}^{r} \frac{r^{2}}{p^{2}} d p d r=(1-\lambda) \frac{1}{4}+\lambda\left[\frac{1}{2} \int_{0}^{1} r d r\right]=\frac{1}{4},
\end{aligned}
$$


which is invariant with $\lambda$. As for firm $B$, we have

$$
\left.\Pi_{B}(\lambda)\right|_{\lambda \geq 8 / 9}=\lambda \frac{1}{2} \int_{0}^{1} \int_{\frac{r}{2}}^{r} p(1-G(p)) g(p) d p d r=\lambda \frac{1}{2} \int_{0}^{1} \int_{\frac{r}{2}}^{r} r \frac{r-p}{p^{2}} d p d r=\lambda \frac{1}{4}(1-\ln 2),
$$

which is an increasing function of $\lambda$ (as $\ln 2<1$ ).

\subsection{Proof of the result in Example 5.4}

Personalized prices. Note first that the second stage equilibrium prices are still characterized by Proposition 3

$$
G^{x}(p)= \begin{cases}\frac{p-(1-\beta) p_{B}^{\prime x}}{\beta p} & \text { for } p \in\left[(1-\beta) p_{B}^{\prime x}, p_{B}^{\prime x}\right] \\ 0 & \text { otherwise. }\end{cases}
$$

Where $p_{B}^{\prime x} \equiv \min \left\{p_{A}, p_{B}, r(x)\right\}$. Given any uniform prices $p_{A}$ and $p_{B}$, the expected payoff from personalized pricing is equal to $\tilde{\pi}=(1-\beta) p_{B}^{\prime x}$. Note that we assume that in stage one, no firm will charge more than $\zeta$ and hence that $p_{B}^{\prime x} \equiv \min \left\{p_{A}, p_{B}, r(x)\right\}=$ $\min \left\{p_{A}, p_{B},\right\}$.

Uniform prices. Note that in any subgame-perfect mixed strategy Nash equilibrium, players must choose prices from the same interval and only the upper bound of the interval can be assigned positive mass by at most one firm. No firm wants to charge a price below the lower bound of the interval since this decreases both first stage and second stage expected profits. No firms wants to charge a price above the upper bound of the interval since this will guarantee zero profits on anonymous consumers and does not affect expected profits obtained from personalized pricing. Now take any price $p$ in the interval different from the upper bound and suppose that at least one firm, say firm $A$, plays this price with positive probability. Firm $B$ should be indifferent between playing price $p$ and any other price $p^{\prime}$ in the interval. However, since firm $A$ plays $p$ with positive probability, firm $B$ will always find a price $p^{\prime}=p-\epsilon$ that will guarantee higher profits: instead of sharing the consumers at this price $p$ it can sell to the consumer for sure by slightly undercutting price $p$. However, it cannot be firm $B$ that plays the upper bound, $p=\zeta$ with positive probability. The expected payoff to firms $A$ and $B$ from setting a uniform price equal to $p$ is equal to:

$$
\begin{aligned}
\Pi_{A}^{e}(p)= & \lambda\left\{\nabla_{B}\left(\int_{\underline{p}}^{p}(1-\beta) \rho h_{B}(\rho) d \rho+(1-\beta) p\left(1-H_{B}(p)\right)\right)+\left(1-\nabla_{B}\right)(1-\beta) p\right\} \\
& +(1-\lambda) p\left[1-\nabla_{B}+\nabla_{B}\left(1-H_{B}(p)\right)\right], \\
\Pi_{B}^{e}(p)= & \beta \lambda\left\{\nabla_{A}\left(\int_{\underline{p}}^{p}(1-\beta) \rho h_{A}(\rho) d \rho+(1-\beta) p\left(1-H_{A}(p)\right)\right)+\left(1-\nabla_{A}\right)(1-\beta) p\right\} \\
& +(1-\lambda) p\left[1-\nabla_{A}+\nabla_{A}\left(1-H_{A}(p)\right)\right] .
\end{aligned}
$$

The first term is the expected payoff from personalized pricing given that the other firm follows the strategies as defined in the proposed mixed strategy equilibrium. The second term is the expected profits to be obtained from consumers it will not recognize given that the other firm follows the strategies as defined in the proposed mixed strategy equilibrium. 
Suppose first that $\nabla_{A}<1$ and hence that $\nabla_{B}=1$. Now consider the expected payoff to firm $A$ from setting a uniform price equal to $p \in[p, \zeta)$ is equal to:

$$
\Pi_{A}^{e}(p)=\lambda\left\{\int_{\underline{p}}^{p}(1-\beta) \rho h_{B}(\rho) d \rho+(1-\beta) p\left(1-H_{B}(p)\right)\right\}+(1-\lambda) p\left[1-H_{B}(p)\right] .
$$

It must be that firm $A$ is indifferent between any $p$ in the interval $[\underline{p}, \zeta)$. We hence have that $\partial \Pi_{A}^{e}(p) / \partial p=0$ for all $p \in[\underline{p}, \zeta)$ :

$$
(1-\lambda) p h_{B}(p)-\left(1-H_{B}(p)\right)(1-\lambda+(1-\beta) \lambda)=0 .
$$

The explicit solution of this differential equation is $H_{B}(p)=c p^{-\frac{1-\lambda \beta}{1-\lambda}}+1$. But then it must be that $H_{B}(\zeta)=1=c \zeta^{-\frac{1-\lambda \beta}{1-\lambda}}+1$ and hence that $c=0$, a contradiction.

Suppose now that $\nabla_{B}<1$ and hence that $\nabla_{A}=1$. Then, the expected payoff to firm $B$ from setting a uniform price equal to $p \in[\underline{p}, \zeta)$ is equal to:

$$
\Pi_{B}^{e}(p)=\beta \lambda\left\{\int_{\underline{p}}^{p}(1-\beta) \rho h_{A}(\rho) d \rho+(1-\beta) p\left(1-H_{A}(p)\right)\right\}+(1-\lambda) p\left[1-H_{A}(p)\right] .
$$

It must be that firm $B$ is indifferent between any $p$ in the interval $[\underline{p}, \zeta)$. We hence have that $\partial \Pi_{B}^{e}(p) / \partial p=0$ for all $p \in[\underline{p}, \zeta)$ :

$$
(1-\lambda) p h_{A}(p)-\left(1-H_{A}(p)\right)(1-\lambda+(1-\beta) \beta \lambda)=0 .
$$

The explicit solution of this differential equation is $H_{A}(p)=c p^{-\frac{1-\lambda+(1-\beta) \beta \lambda}{1-\lambda}}+1$. But then it must be that $H_{A}(\zeta)=1=c \zeta^{\frac{1-\lambda+(1-\beta) \beta \lambda}{1-\lambda}}+1$ and hence that $c=0$, again a contradiction. 